\title{
Behavioral and Single-Neuron Sensitivity to Millisecond Variations in Temporally Patterned Communication Signals
}

\author{
C.Christa A. Baker, Lisa Ma, @Chelsea R. Casareale, and @Bruce A. Carlson \\ Department of Biology, Washington University in St. Louis, St. Louis, Missouri 63130-4899
}

In many sensory pathways, central neurons serve as temporal filters for timing patterns in communication signals. However, how a population of neurons with diverse temporal filtering properties codes for natural variation in communication signals is unknown. Here we addressed this question in the weakly electric fish Brienomyrus brachyistius, which varies the time intervals between successive electric organ discharges to communicate. These fish produce an individually stereotyped signal called a scallop, which consists of a distinctive temporal pattern of $\sim 8-12$ electric pulses. We manipulated the temporal structure of natural scallops during behavioral playback and in vivo electrophysiology experiments to probe the temporal sensitivity of scallop encoding and recognition. We found that presenting time-reversed, randomized, or jittered scallops increased behavioral response thresholds, demonstrating that fish's electric signaling behavior was sensitive to the precise temporal structure of scallops. Next, using in vivo intracellular recordings and discriminant function analysis, we found that the responses of interval-selective midbrain neurons were also sensitive to the precise temporal structure of scallops. Subthreshold changes in membrane potential recorded from single neurons discriminated natural scallops from time-reversed, randomized, and jittered sequences. Pooling the responses of multiple neurons improved the discriminability of natural sequences from temporally manipulated sequences. Finally, we found that single-neuron responses were sensitive to interindividual variation in scallop sequences, raising the question of whether fish may analyze scallop structure to gain information about the sender. Collectively, these results demonstrate that a population of interval-selective neurons can encode behaviorally relevant temporal patterns with millisecond precision.

Key words: animal communication; electrosensation; sensory coding; temporal coding; weakly electric fish

Significance Statement

The timing patterns of action potentials, or spikes, play important roles in representing information in the nervous system. However, how these temporal patterns are recognized by downstream neurons is not well understood. Here we use the electrosensory system of mormyrid weakly electric fish to investigate how a population of neurons with diverse temporal filtering properties encodes behaviorally relevant input timing patterns, and how this relates to behavioral sensitivity. We show that fish are behaviorally sensitive to millisecond variations in natural, temporally patterned communication signals, and that the responses of individual midbrain neurons are also sensitive to variation in these patterns. In fact, the output of single neurons contains enough information to discriminate stereotyped communication signals produced by different individuals.

\section{Introduction}

Timing patterns carry information in the communication signals of a wide range of animals. For instance, the time intervals be-

Received Feb. 27, 2016; revised June 14, 2016; accepted July 5, 2016.

Author contributions: C.A.B. and B.A.C. designed research; C.A.B., L.M., and C.R.C. performed research; C.A.B., L.M., and C.R.C. analyzed data; C.A.B. and B.A.C. wrote the paper.

This work was supported by the National Science Foundation (Grant IOS-1050701 to B.A.C.), the National Institute on Deafness and Other Communication Disorders (Grant F31-DC012452 to C.A.B.), and the McDonnell Center for

Systems Neuroscience (to B.A.C.). We thank Gary D. London for collecting some of the behavioral playback data. The authors declare no competing financial interests.

Correspondence should be addressed to Bruce A. Carlson, Washington University in St. Louis, Department of Biology, Campus Box 1137, One Brookings Drive, St. Louis, M0 63130-4899. E-mail: carlson.bruce@wustl.edu.

DOI:10.1523/JNEUROSCI.0648-16.2016

Copyright $\odot 2016$ the authors $\quad 0270-6474 / 16 / 368985-16 \$ 15.00 / 0$ tween successive sound or light pulses are species-specific in certain frogs and insects (Lloyd, 1966; Ewing and Bennet-Clark, 1968; Loftus-Hills and Littlejohn, 1971; Pollack, 2000). Previous studies have shown that circuits process these types of temporal patterns with central neurons that are selective for particular timing intervals (Rose and Capranica, 1983; Grothe, 1994; Fortune and Rose, 1997a; Edwards et al., 2002; Sakai et al., 2009; Pluta and Kawasaki, 2010; Goel and Buonomano, 2014). These response properties arise from synaptic mechanisms such as temporal summation and short-term synaptic plasticity (Fortune and Rose, 2000; George et al., 2011; Rose et al., 2011; Baker and Carlson, 2014), as well as from intrinsic postsynaptic membrane properties (Fortune and Rose, 1997b; Trussell, 1999; Fortune and Rose, 2003; Carlson and Kawasaki, 2006; Kohashi and Carlson, 
2014). Although we have a good understanding of how circuits can establish single-neuron temporal selectivity, how a population of neurons with a diverse range of temporal filtering properties contributes to the detection and discrimination of natural timing patterns is unknown.

Here we investigate how a population of interval-selective midbrain neurons mediates the detection of temporally patterned communication signals. We use mormyrid weakly electric fish because the timing patterns in their communication signals have clear behavioral relevance, and because interval-selective neurons are easily accessible for in vivo electrophysiological recordings (Carlson, 2009; Baker and Carlson, 2014). Further, electric communication signals are easy to record, manipulate, and play back, such that we can provide identical stimuli during in vivo recordings and during experiments in freely behaving fish.

Mormyrids vary the time intervals between successive electric organ discharges (EODs) to communicate (for review, see Carlson, 2002). At least three quantitatively distinct temporal patterns of pulses have been linked to different social contexts in our study species, Brienomyrus brachyistius (Carlson and Hopkins, 2004). All three of these signals consist of a rapid decrease followed by a gradual increase in interpulse intervals (IPIs). One signal in particular, called a scallop, consists of individually distinctive IPI sequences (Serrier and Moller, 1989; Carlson and Hopkins, 2004). Scallops therefore present a valuable opportunity to test the specificity of temporal pattern processing by a sensory pathway.

Electric communication signals are processed by a dedicated sensory pathway (Xu-Friedman and Hopkins, 1999; Baker et al., 2013). Sensory receptors called knollenorgans fire a single, timelocked spike in response to each EOD (Bennett, 1965). Afferents relay these spikes to the hindbrain nucleus of the electrosensory lateral line lobe (nELL), where corollary discharge inhibition blocks responses to the fish's own EODs (Bell and Grant, 1989). Axons from nELL project to the anterior exterolateral nucleus (ELa) of the midbrain torus semicircularis. ELa sends its only output to the posterior exterolateral nucleus (ELp), where sensitivity to interpulse intervals arises (Carlson, 2009). ELp neurons transform the temporal code for IPI into a population code, with neurons exhibiting a wide diversity of interval selectivity (Carlson, 2009; George et al., 2011; Baker et al., 2013; Ma et al., 2013; Baker and Carlson, 2014; Kohashi and Carlson, 2014).

In the present study, we measure behavioral and singleneuron responses to natural scallops and manipulated versions of scallops to understand the temporal precision of signal detection. We demonstrate that fish are behaviorally sensitive to millisecond variations in natural timing patterns, and we show that the responses of interval-selective midbrain neurons contain enough information to resolve natural patterns from these variations. Further, the responses of single neurons can be used to distinguish scallop patterns produced by different individuals, suggesting that scallops could be used to determine sender identity.

\section{Materials and Methods}

Animals. Eighty-one B. brachyistius fish ranging from 5.0 to $9.2 \mathrm{~cm}$ in standard length of both sexes contributed data to this study. Fish were housed in single-species tanks with a $12 \mathrm{~h}$ light/dark cycle, water conductivity of $200-$ $400 \mu \mathrm{S} / \mathrm{cm}$, and water temperature of $25-29^{\circ} \mathrm{C}$. We fed the fish live black worms four times per week. Fish were obtained through the aquarium trade. All procedures were carried out in accordance with National Institutes of Health guidelines and were approved by the Institutional Animal Use and Care Committee at Washington University in St. Louis.

Scallop stimulus generation. We chose six scallops as standard stimuli: one scallop from each of three males, and one scallop from each of three females (Fig. 1A). These stimuli were taken from scallops recorded in a previous study (Carlson and Hopkins, 2004). Four of these scallops consisted of 11 IPIs (F1, F2, M2, M3), one consisted of 14 IPIs (F3), and one consisted of 10 IPIs (M1). To test the sensitivity of scallop detection to precise temporal patterning, we generated the following five temporal manipulations of natural scallops: time-reversed sequences, in which the IPI order of the natural scallop was reversed; randomized sequences, in which the IPI order was randomly shuffled on each stimulus presentation; and three sequences with jitter added to the timing of each pulse. For randomized stimuli, we randomized the IPI order on each stimulus presentation to allow the recording of responses to stimuli that preserved the overall IPI distribution but not the temporal order of IPIs. This allowed us to test responses to randomization in general, instead of testing responses to one particular randomized sequence. We generated sequences with 1,3 , or $5 \mathrm{~ms}$ of jitter by adding a value drawn randomly from a Gaussian distribution with a mean of 0 and an SD of 1,3 , or $5 \mathrm{~ms}$, respectively. We added an independently drawn jitter value to each pulse in the sequence on each stimulus presentation, such that jitter values were independent across pulses within a stimulus, and across stimulus presentations. We jittered each stimulus presentation independently to test responses to jitter in general, instead of testing responses to one particular jittered sequence. We also generated start-shifted versions of scallops, in which we systematically varied the starting IPI and looped from the end of the natural sequence to the beginning to maintain the same number of pulses with the same overall IPI distribution. For instance, in a scallop with 11 IPIs, one start-shifted stimulus consisted of the 2nd through the 11th IPIs followed by the 1st IPI. Another stimulus consisted of the 3rd through the 11th IPIs followed by the 1st and 2nd IPIs, and so on for all possible versions.

To relate natural variation in scallop patterns to our jittered, timereversed, and randomized manipulations, we computed jitter values between the four natural scallops with the same number of IPIs. Following the procedure used to create jittered stimuli, we defined jitter as the SD of the difference in corresponding EOD times for each pair of scallops. We defined the time of the first EOD in each scallop as $0 \mathrm{~ms}$. We repeated this procedure for all six natural scallops, and their respective time-reversed and randomized sequences. Since the natural scallops in our dataset contained $10-14$ IPIs, there were $3.6 \times 10^{6}$ to $8.7 \times 10^{10}$ possible randomized sequences for each scallop. Due to computational resource limitations, we calculated the jitter between 100,000 randomly selected randomized sequences and each natural scallop.

Behavioral playback experiments. To test behavioral responses to scallops, we recorded the EOD output of a fish during the presentation of natural and manipulated scallop sequences using previously described methods (Carlson et al., 2011; Lyons-Warren et al., 2012). Briefly, we placed a rectangular plastic chamber $(4.1 \times 4.1 \times 20.3 \mathrm{~cm})$ in the middle of the home tank of a singly housed fish $(20.3 \times 25.4 \times 40.6 \mathrm{~cm})$. Most fish entered the chamber voluntarily within $\sim 5 \mathrm{~min}$. If fish were not within the chamber after 5 min, we guided fish into the chamber with a net. Netted caps were placed over each end of the chamber to keep the fish inside during the experiment. Fish were then allowed an additional 10 min to acclimate to the chamber. A pair of $\mathrm{Ag} / \mathrm{AgCl}$ electrodes oriented horizontally on both sides of the chamber delivered the stimulus, and a pair of $\mathrm{Ag} / \mathrm{AgCl}$ electrodes oriented vertically on both ends of the chamber recorded the EOD output of the fish. We delivered 20 repetitions of a single scallop stimulus consisting of bipolar square electric pulses of $2 \mathrm{~ms}$ duration, with $30 \mathrm{~s}$ between stimulus presentations to reduce habituation. We randomized stimulus order within and across fish. All stimuli were generated in Matlab 7 (MathWorks), digital-toanalog converted at a rate of $97.7 \mathrm{kHz}$ (RX8, Tucker-Davis Technologies), and attenuated (PA5, Tucker-Davis Technologies) before delivery to an analog stimulus isolator (Model 2200, A-M Systems) connected to the stimulus electrodes.

To record the EOD output of a fish, we amplified electrical activity 100 times with bandpass filtering $(0.1 \mathrm{~Hz}-20 \mathrm{kHz}$; Model 1700, A-M Systems), recorded EOD times as events that crossed a manually set threshold, and then saved these times using custom software in Matlab. We computed the spike density function (SDF) by convolving each EOD time of occurrence with a Gaussian of 200 ms width (Carlson and Hop- 
A
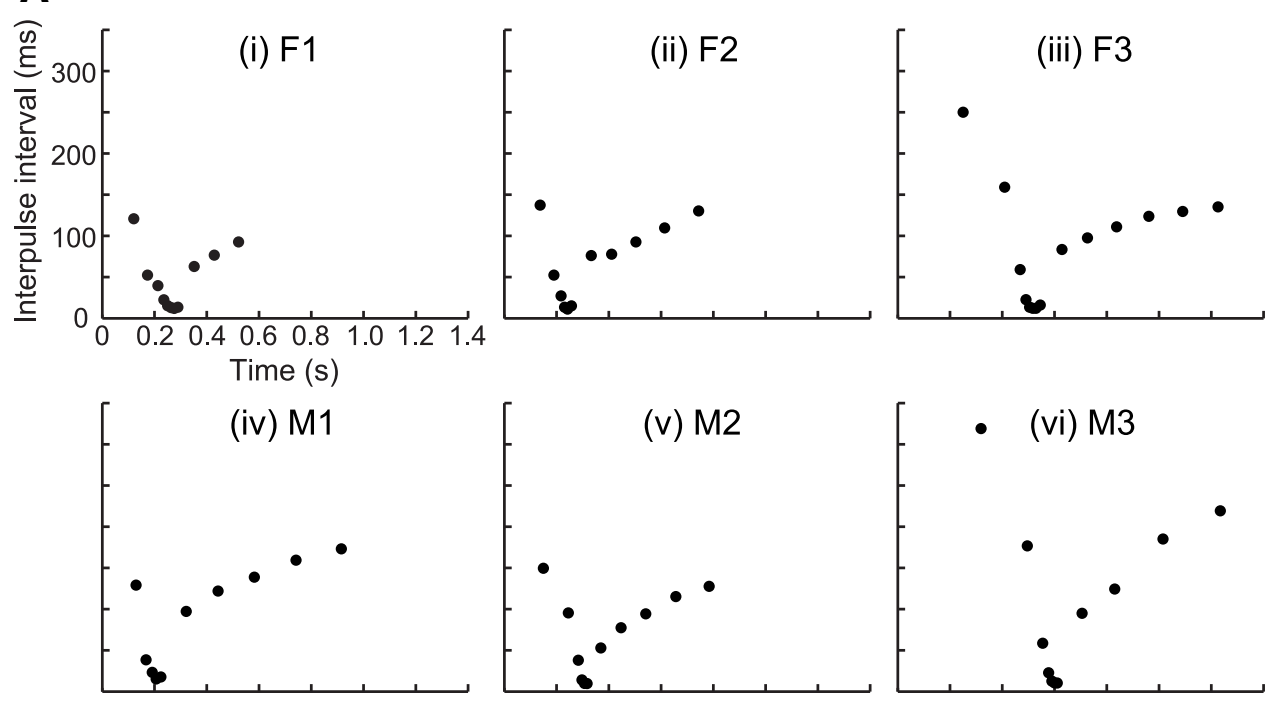

- (vi) M3

B

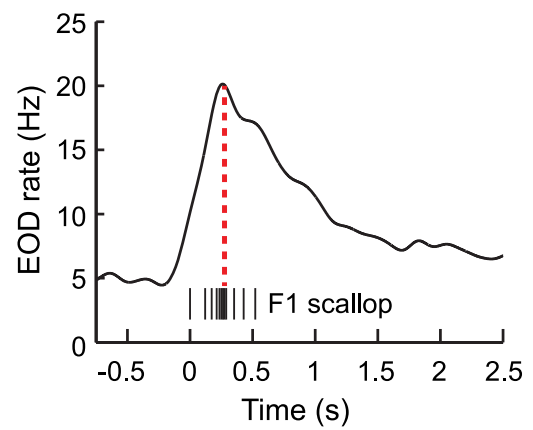

C

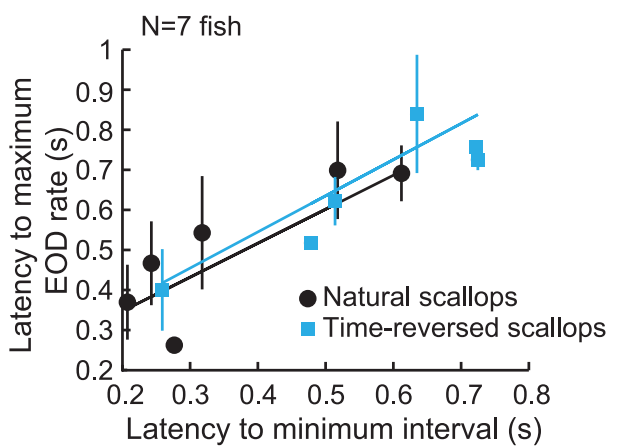

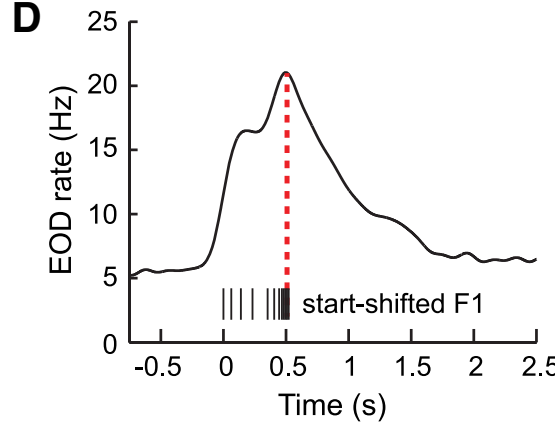

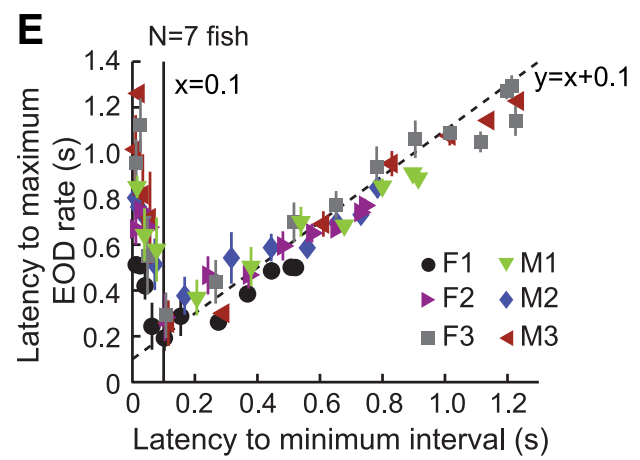

Figure 1. The timing of behavioral responses is sensitive to scallop temporal structure. $A$, Our library of natural scallop patterns consisted of one sequence recorded from each of three females (F1-F3) and from each of three males (M1-M3). Each point represents the IPI vs time of each electric pulse in the scallop. $\boldsymbol{B}$, EOD rate recorded from one fish in response to a scallop (F1) presented at time $=0 \mathrm{~s}$. Tick marks below the EOD rate curve represent the time of each pulse in the stimulus. The red vertical line represents the time of the minimum IPI in the stimulus. $C$, Latency to the maximum EOD rate of the fish vs latency to the minimum IPI of the stimulus for natural and time-reversed scallops. Each point represents the average across seven fish in response to each natural scallop in $\boldsymbol{A}$. Error bars represent SEM. Lines are the results of a linear regression (natural scallops: $\boldsymbol{r}^{2}=0.72, p=0.033$; time-reversed scallops: $\boldsymbol{r}^{2}=0.81, p=0.014$ ). $\boldsymbol{D}$, Same as $\boldsymbol{B}$ for a start-shifted version of the F1 scallop. This stimulus consisted of the 9th through 11th IPIs of the F1 scallop followed by the 1st through 8th IPIs. $E$, The latency to the maximum E0D rate of the fish vs the latency to the minimum IPI for start-shifted sequences of all six natural scallops. Each point represents the average across seven fish, and error bars represent the SEM. Solid line represents $x=0.1$, and dotted line represents $y=x+0.1$.

kins, 2004), and then averaging over stimulus presentations. We measured the baseline EOD rate of the fish by averaging the SDF over a window starting $0.2 \mathrm{~s}$ after the start of a $5 \mathrm{~s}$ prestimulus period and ending $0.2 \mathrm{~s}$ before stimulus onset.

To investigate how fish responded to natural and start-shifted scallop stimuli, we measured the maximum EOD rate that occurred in a window starting $0.1 \mathrm{~s}$ before stimulus onset up to $2.1 \mathrm{~s}$ after stimulus offset. The response window started before stimulus onset since the Gaussian used in the convolution was symmetric in time, such that a response immediately following stimulus onset could affect the SDF for up to $100 \mathrm{~ms}$ (i.e., half-width of the Gaussian) before the stimulus. We measured response latency relative to stimulus onset. In practice, the response latency was never negative. To measure behavioral response thresholds, we defined a behavioral response from the fish as a maximum EOD rate exceeding the prestimulus mean +3 SDs that occurred between $0.1 \mathrm{~s}$ before stimulus onset up to $2.1 \mathrm{~s}$ after stimulus offset. For each stimulus, we varied the intensity until we found the weakest intensity (to the nearest decibel) to which the fish responded. We defined this intensity as the behavioral response threshold. The actual threshold stimulus intensity (in millivolts per centimeter) was determined using a calibration curve obtained by 
recording in the center of the playback chamber. We normalized threshold intensities in response to manipulations of a natural scallop to the threshold intensity for the natural scallop, and then averaged across the six scallop stimuli to get a single data point for each manipulation for each fish.

Whole-cell recordings. We prepared fish for in vivo recordings from ELp as described previously (Carlson, 2009; Lyons-Warren et al., 2013; Baker and Carlson, 2014). Briefly, fish were anesthetized in $300 \mathrm{mg} / \mathrm{L}$ tricaine methanesulfonate (MS-222) and paralyzed with an intramuscular injection of $100 \mu \mathrm{l}$ of $0.1 \mathrm{mg} / \mathrm{ml}$ gallamine triethiodide (Flaxedil). We positioned the fish on a platform with lateral supports and respirated the fish with $100 \mathrm{mg} / \mathrm{L}$ MS-222 during surgery. We placed a drop of $0.4 \%$ lidocaine on the skin overlying the incision site, and then made an incision to uncover the skull overlying ELp. Next we glued a head post to the skull before using a dental drill and forceps to remove a rectangular piece of skull covering ELp. We placed the ground electrode on the nearby cerebellum. Following surgery, we switched respiration to fresh water and allowed the fish to recover from anesthesia. We monitored the anesthetized state of the fish with a pair of electrodes oriented parallel to its electric organ to record the fictive EOD produced by the electromotor neurons (Carlson, 2009; Lyons-Warren et al., 2013). Anesthesia silences the fictive EOD, such that the return of fictive discharges indicates that the fish has recovered from anesthesia. At the end of the recording session, the respiration of the fish was switched back to $100 \mathrm{mg} / \mathrm{L} \mathrm{MS}-222$ until no fictive EOD could be recorded, and then the fish was killed by freezing.

We obtained intracellular, whole-cell patch recordings in currentclamp using previously published methods (Rose and Fortune, 1996; Carlson, 2009; Baker and Carlson, 2014). Briefly, we used a Flaming/ Brown micropipette puller (Model P-97, Sutter Instruments Co.) to fabricate glass patch micropipettes with resistances of 20-40 M $\Omega$. We backfilled the pipette tip with a solution containing the following (in $\mathrm{mm}$ ): $100 \mathrm{CH}_{3} \mathrm{CO}_{2} \mathrm{~K}, 2 \mathrm{KCl}, 1 \mathrm{MgCl}, 5$ EGTA, 10 HEPES, $20 \mathrm{KOH}$, and 43 biocytin. We filled the pipette shank with the same solution, except that we replaced biocytin with D-mannitol (Carlson, 2009; Baker and Carlson, 2014). Initial seals were $>1 \mathrm{G} \Omega$. Recordings were amplified 10 times and low-pass filtered (cutoff frequency, $10 \mathrm{kHz}$ ) using an Axopatch 200B amplifier (Molecular Devices), digitized at a rate of $97.7 \mathrm{kHz}$ (Model RX8 Digitizer, Tucker Davis Technologies), and saved using custom software written in Matlab 7.

We delivered electrosensory stimulation using electrodes positioned around the perimeter of the recording chamber. Three vertically oriented electrodes on both sides of the chamber delivered stimulation transverse to the fish. Two vertically oriented electrodes at the front and back of the chamber delivered stimulation longitudinal to the fish (Lyons-Warren et al., 2013). We used stimuli consisting of monopolar square electric pulses.

After obtaining a recording, we first varied the duration $(0.2-2 \mathrm{~ms})$, intensity $(3-71 \mathrm{mV} / \mathrm{cm})$, polarity (normal or reversed), and stimulus orientation (transverse or longitudinal to the fish) of a single pulse to elicit maximal postsynaptic potential (PSP) amplitudes from each neuron. All subsequent stimuli delivered during a recording then used these parameters while varying only the IPIs (Carlson, 2009; Baker and Carlson, 2014). We discarded responses to stimulus repetitions in which stimulus pulses occurred within $2-5 \mathrm{~ms}$ after a fictive EOD, since corollary discharge inhibition in the hindbrain blocks sensory responses within this window (Bell and Grant, 1989).

Data analysis. We removed spikes from recording traces and measured PSP amplitudes as described previously (Baker and Carlson, 2014). Briefly, to remove spikes, we first defined the spike start as the point where the smoothed (moving average filter with width of $0.5 \mathrm{~ms}$ ) derivative of the recorded potential first exceeded the prestimulus mean +4 SDs, and spike end as the point where the smoothed derivative decreased below the prestimulus mean $-1 \mathrm{SD}$. If the smoothed derivative did not meet our spike end criterion, we instead defined the spike end as the first minimum in the smoothed derivative up to $8 \mathrm{~ms}$ after the spike start. We measured the resting potential (RP) of the neuron by first averaging the spike-removed traces over 10 presentations of the same stimulus, and then averaging the potential over a $50 \mathrm{~ms}$ window immediately preceding the stimulus. We measured PSP amplitudes by subtracting the RP from the maximum potential (after spike removal) that occurred between $3 \mathrm{~ms}$ after each stimulus pulse up to the time of the next pulse. For presentation purposes, we removed the stimulus artifact from recordings by linearly extrapolating the potential between the time of pulse onset to $0.5 \mathrm{~ms}$ after pulse offset.

We measured the membrane time constant $\left(\tau_{m}\right)$ and input resistance $\left(R_{m}\right)$ by fitting the response of each neuron to a $100 \mathrm{~ms},-0.10 \mathrm{nA}$ current pulse with a double exponential function, as previously described (Gittelman et al., 2009; Baker and Carlson, 2014). We then estimated membrane capacitance $\left(C_{m}\right)$ according to the following:

$$
C_{m}=\frac{\tau_{m}}{R_{m}}
$$

Next we determined IPI tuning by collecting responses to scanning IPI stimuli consisting of IPIs ranging from 10 to $200 \mathrm{~ms}$, and generating IPI tuning curves as previously described (Baker and Carlson, 2014). Briefly, we normalized PSP amplitudes in response to each IPI by the maximum PSP amplitude. If the normalized amplitudes to all IPIs were $>0.85$, we classified the neuron as all-pass (i.e., no IPI tuning). Otherwise, we used sigmoidal and Gaussian fits to the tuning curves to classify IPI tuning as high-pass, low-pass, bandpass, or bandstop (for details, see Baker and Carlson, 2014). If the tuning of a neuron did not fit any of these classes, we classified it as complex.

We next collected responses to natural and manipulated scallop stimuli. For each neuron, we randomly selected a natural scallop from our library. We then presented 10 repetitions each of the natural sequence; a time-reversed sequence; randomized sequences; and sequences with 1,3 , and $5 \mathrm{~ms}$ of added jitter. We randomized stimulus presentation order. Next, we presented 10 additional repetitions of the natural scallop sequence. We repeated this procedure with another randomly selected scallop until we had presented all manipulations of all six scallops, or until we lost the recording. We measured the PSPs evoked by each stimulus pulse on each repetition.

Inclusion criteria included the following: (1) the RP remained below $-40 \mathrm{mV}$ throughout the recording; and (2) the RP varied $\leq 5 \mathrm{mV}$ throughout the recording. Fifty-six of 60 neurons fit these criteria. Two of the excluded neurons were high-pass and two were low-pass. The RP, membrane capacitance, and membrane time constant of the four excluded neurons were within the range of those of the included neurons. For the 56 included neurons, there were no differences in RP (one-way ANOVA, $F_{(3)}=0.68, p=0.57$; mean $=-57.3 \pm 0.4 \mathrm{mV}$ ), membrane capacitance (one-way ANOVA, $F_{(3)}=1.1, p=0.36$; mean $=86.7 \pm 10.2$ $\mathrm{pF}$ ), or membrane time constant (one-way ANOVA, $F_{(3)}=0.46 \mathrm{~m} p=$ 0.71 ; mean $=12.0 \pm 1.2 \mathrm{~ms}$ ) across IPI tuning groups.

Discriminant function analysis. To assess the degree to which the responses of single ELp neurons could be used to distinguish among natural and manipulated scallop sequences, we used discriminant function analysis (DFA). DFA defines a set of functions to group multivariate data by maximizing intergroup variance while minimizing intragroup variance (Cooley and Lohnes, 1971). These functions can then be used to assign independent data to one of the predefined groups. We used the PSPs evoked by the second through the last pulse in each stimulus as variables, and we used the PSPs measured on each stimulus presentation as a single observation. Occasionally, our method of adding jitter resulted in a negative IPI (i.e., the jitter value to add was more negative than -1 times the natural IPI). This happened almost exclusively for $5 \mathrm{~ms}$ jitter sequences. In these cases, we threw out the responses of the neuron to that particular stimulus repetition. Thus, we had 10 observations each for natural, time-reversed, randomized, $1 \mathrm{~ms}$ jitter-added, and $3 \mathrm{~ms}$ jitteradded sequences, and 5-10 observations for $5 \mathrm{~ms}$ jitter-added sequences in all neurons. We used the manipulation type as the grouping variable. We then repeated 10 presentations of the natural sequence to determine the probability that natural sequences were classified as such. To minimize the possibility of long-term changes in responses affecting classification, we used five randomly selected repetitions from the first set of responses to natural scallops and five randomly selected repetitions from the repeated natural scallop to define the DFA. We then used these dis- 
criminant functions to classify responses to each of the 10 remaining repetitions of the natural scallop. To determine the discriminability of spiking responses, we performed DFA using the number of spikes in response to each stimulus pulse instead of PSP amplitude.

To determine whether ELp responses are sensitive to individual variation in scallops, we performed DFA on the responses of a neuron to the four natural scallops in our library that had the same total number of IPIs. We used scallop ID as the grouping variable. For this analysis, we used the responses of only those neurons from which we recorded responses to all four natural scallops as well as responses to repeated presentations of all four scallops. For each scallop, we randomly selected five responses from each of the two repeated blocks of natural stimuli to define the discriminant functions. We used the remaining 10 responses to the natural scallop to test classification accuracy.

To implement DFA, we used a built-in discriminant function classifier (ClassificationDiscriminant.m) in Matlab that assumes a Gaussian mixture distribution. We also used a built-in function (mahal.m) to calculate Mahalanobis distances between each of 10 responses to the repeated natural sequence and the centroid of each manipulation group. This method assumes that the responses have similar distributions and variance. To assess the discriminability of responses to natural versus timereversed, randomized, or jittered sequences, we assigned each of 10 test responses to the group (e.g., natural or time reversed) with the smallest Mahalanobis distance. To study the discriminability of responses to natural sequences from different individuals, we assigned each of 10 test responses to the group with the smallest Mahalanobis distance. We divided the number of repetitions assigned to each group by the total number of repetitions to get the probability of classification for each manipulation.

Next, we investigated how classification performance may be affected by pooling the responses of multiple neurons together. From the singleneuron classification probabilities, we calculated group probabilities according to the following:

$$
\sum_{i} \prod_{k} p(i \mid k)
$$

where $p(i \mid k)$ is the probability that the responses of neuron $i$ are classified as stimulus $k$. If we recorded responses from the same neuron to multiple natural scallops and manipulations, we averaged the classification probabilities across the natural scallops. We calculated group classification probabilities for combinations of two to 10 neurons in the following instances: (1) natural versus time-reversed sequences; (2) natural versus randomized sequences; (3) natural versus $1 \mathrm{~ms}$ jitter-added sequences; (4) natural versus $3 \mathrm{~ms}$ jitter-added sequences; and (5) natural versus 5 $\mathrm{ms}$ jitter-added sequences. For pairs of neurons, we calculated the group probabilities for all possible combinations. Because calculating the joint probabilities for all possible combinations of more than five neurons was computationally time-intensive, we instead calculated the probabilities for 10,000 randomly selected combinations of 3 to 10 neurons. The SEs of these measurements were $<0.3 \%$ of the means, indicating that our random samples were representative of all possible combinations. We calculated group classification probabilities for all possible combinations of 2 to 10 neurons in the following instances: (1) two different natural scallops; (2) three different natural scallops; and (3) four different natural scallops. For classification accuracies that did not reach 1 at the maximum group size, we fit the classification accuracy versus group size with a logarithmic function according to the following:

$$
y=a \ln (x)+b
$$

where $y$ is the classification probability, $x$ is the group size, and $a$ and $b$ are constants.

Statistics. All statistical tests were performed using Statistica (StatSoft). Parametric tests included single-sample $t$ tests, paired $t$ tests, one-way ANOVA, and repeated-measures ANOVA. Nonparametric tests included Spearman's rank test. The significance level for all tests was $p<0.05$.

\section{Results}

\section{Fish are behaviorally sensitive to the temporal structure of scallops}

To determine whether the temporal structure of the stereotyped scallop signal was behaviorally significant, we performed playback experiments in which we recorded the electric signaling activity of a fish in response to natural scallops and various temporal manipulations of natural scallops. Our natural stimulus set consisted of one scallop recorded from each of three females, and one scallop recorded from each of three males (Fig. 1A). We measured fish's maximum EOD rates in response to 20 repetitions of each scallop presented at the same intensity $(291 \mathrm{mV} / \mathrm{cm}$; Fig. $1 B$ ). Response strengths depended on the scallop (repeatedmeasures ANOVA, $F_{(5)}=2.7, p=0.039$ ), with fish responding most strongly $($ mean $=20.6 \pm 1.4 \mathrm{~Hz})$ to the scallop consisting of the largest number of IPIs (F3, 14 IPIs) and least strongly (mean $=16.9 \pm 1.4 \mathrm{~Hz})$ to the scallop consisting of the smallest number of IPIs (M1, 10 IPIs). There was no difference in the maximum EOD rates elicited by the four scallops with the same number of IPIs (repeated-measures ANOVA, $F_{(3)}=0.26, p=$ 0.85 ), suggesting that the difference in response strengths was due to different numbers of IPIs in the natural scallops instead of slight differences in the temporal structure of each scallop.

The latency of the maximum EOD rate of a fish depended on the scallop (repeated-measures ANOVA, $F_{(5)}=5.5, p=0.011$ ) and was positively correlated with the latency of the minimum IPI in each scallop (linear regression, $r^{2}=0.72, p=0.033$; Fig. $1 C$, black points). A similar relationship held for responses to time-reversed sequences of natural scallops (linear regression, $r^{2}=0.81, p=0.014$; Fig. $1 C$, blue points). These results suggest that fish are able track the fine temporal structure of the scallop.

We further tested the relationship between the scallop timing pattern and fish's responses by presenting start-shifted versions of natural scallops. Start-shifted versions contained the same IPIs as the natural scallop but varied the starting point. For instance, for a scallop consisting of 11 IPIs, one start-shifted version consisted of the 2 nd through the 11th IPIs followed by the 1st IPI. If the responses of a fish indeed depend on when the minimum IPI occurs in the signal, we should observe a relationship between the latency of the minimum IPI and the latency of the maximum EOD rate of the fish. We recorded the time of the maximal discharge rate of a fish in response to all possible start shifts of all six natural scallops (Fig. 1D). Response latencies were positively correlated with the latencies of the minimum IPI in the stimulus for minimum IPI latencies $>0.1 \mathrm{~s}$ (linear regression, $r^{2}=0.92-0.98, p=$ $0.000001-0.00068$ across all six natural scallops; Fig. $1 E)$. This means that as long the minimum IPI occurred $\sim 100 \mathrm{~ms}$ or later after the start of the sequence, the timing of the responses of the fish tracked the time of the minimum IPI. The response latencies were well fit by the equation $y=x+0.1$ (linear regression, $r^{2}=0.48-0.96$ across all six natural scallops), suggesting that the minimum response latency of the fish is $\sim 100 \mathrm{~ms}$.

Next we sought to probe the importance of precise temporal pattern in determining behavioral responses by measuring changes in EOD rate in response to manipulations of natural scallops. To test how changes in the order of scallop IPIs affected behavioral responses, we generated time-reversed and randomized sequences (Fig. 2A). Randomized sequences were generated independently for each stimulus presentation such that the IPI order could be different on each presentation. We recorded fish's responses to natural, time-reversed, and randomized sequences of all six scallops presented at the same intensity $(291 \mathrm{mV} / \mathrm{cm})$, 


\section{A}

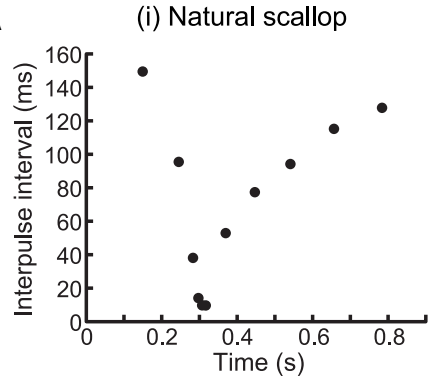

C

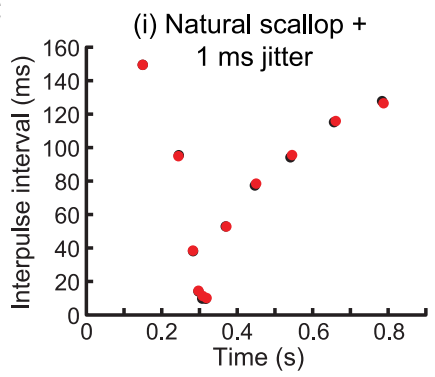

(ii) Time-reversed scallop

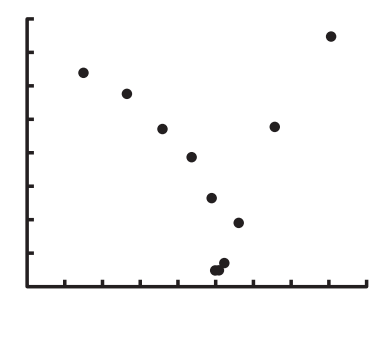

(ii) Natural scallop +

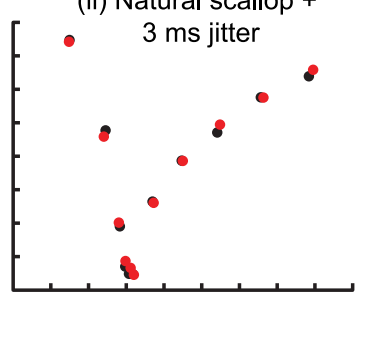

(iii) Randomized scallop

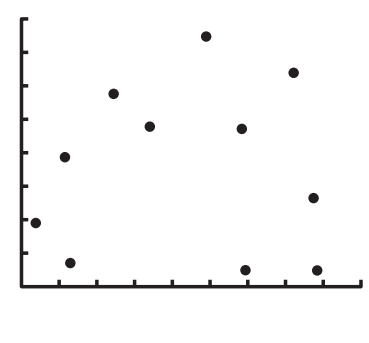

(iii) Natural scallop +

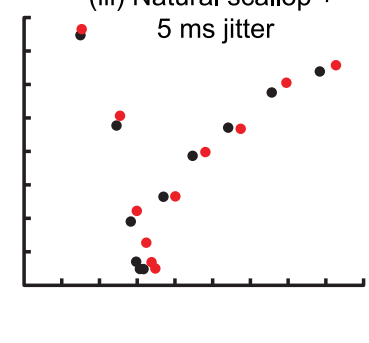

B

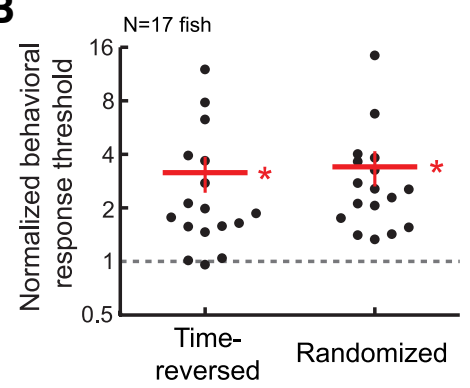

D

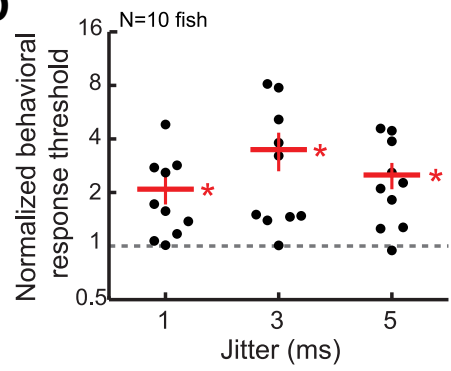

Figure 2. Temporal manipulations of natural scallops increase behavioral response thresholds. $\boldsymbol{A}$, Natural (Ai), time-reversed (Aii), and randomized (Aiii) versions of the M2 scallop. Each point represents the IPI vs time of each electric pulse in the sequence. $\boldsymbol{B}$, Behavioral response thresholds for time-reversed and randomized sequences normalized to the thresholds for natural scallops. Each point represents the average of the normalized thresholds of one fish for manipulations of all six natural scallops. Horizontal bars represent the average across all fish, and error bars represent the SEM. ${ }^{*} p<0.01$, single-sample $t$ test against 1. C, Examples of a natural scallop (M2; black) and one sequence (red) with each of three different amounts of jitter added. $\boldsymbol{D}$, Same as $\boldsymbol{B}$ for jitter-added sequences. ${ }^{*} p<0.02$, single-sample $t$ test against 1 .

and found no differences in the maximum EOD rate in response to the three types of sequences (mean across all sequences $=18.6 \pm 0.2$ $\mathrm{Hz}$; repeated-measures ANOVA, $F_{(2)}=0.72, p=0.51$ ).

To obtain a more sensitive measure of responsiveness, we determined behavioral response thresholds for natural, timereversed, and randomized scallops in 17 fish. We varied stimulus intensity in $1 \mathrm{~dB}$ increments until the fish no longer responded with an increase in EOD rate that exceeded the prestimulus mean EOD rate +3 SDs. The lowest intensity that elicited a response from the fish was defined as the behavioral response threshold.

Behavioral response thresholds for natural scallops ranged from 0.6 to $22.7 \mathrm{mV} / \mathrm{cm}(\mathrm{N}=17$ fish $)$ and were not significantly different across scallops (repeated-measures ANOVA, $F_{(5)}=1.3$, $p=0.30$ ). Because response thresholds were variable within and across fish, we normalized thresholds for time-reversed and randomized sequences to those for natural scallops. Responses to a particular natural scallop and all its manipulations were recorded on the same day. We found that behavioral response thresholds were on average approximately three times higher for timereversed and randomized sequences than for natural sequences ( single-sample $t$ test against 1 ; time-reversed: $t_{(16)}=3.0, p=$ 0.0088 ; randomized: $t_{(16)}=3.1, p=0.0063$; Fig. $\left.2 B\right)$. This means that time-reversed and randomized signals had to be approximately three times more intense than natural signals to elicit a behavioral response. These results reveal that the IPI order, and not just the overall IPI composition, of natural scallops is relevant for fish's responses.

To determine the extent to which the system is tuned to the precise temporal structure of scallops, we recorded behavioral responses to natural scallop sequences with jitter added to each pulse. To do so, we randomly drew a value from a Gaussian distribution with a mean of 0 and SDs of 1,3 , or $5 \mathrm{~ms}$, and added this value to the time of each pulse in the natural scallop (Fig. 2C). Jitter values were added independently to each pulse in a stimulus during each presentation such that the jittered stimulus was slightly different on each presentation. The overall effect resulted in IPIs that were sometimes shorter and sometimes longer than those in the natural scallop.

We first recorded behavioral responses to natural and jittered sequences of all six scallops presented at the same intensity (291 $\mathrm{mV} / \mathrm{cm}$ ). There were no differences in the maximum EOD rate in response to natural, $1 \mathrm{~ms}$ jitter-added, $3 \mathrm{~ms}$ jitter-added, and $5 \mathrm{~ms}$ jitter-added sequences (mean across all sequences $=18.7 \pm 0.2$ $\mathrm{Hz}$; repeated-measures ANOVA, $\left.F_{(3)}=0.98, p=0.42\right)$. Next, we measured behavioral response thresholds to natural and jitteradded sequences. Thresholds to natural scallops ranged from 0.6 to $6.2 \mathrm{mV} / \mathrm{cm}(\mathrm{N}=10 \mathrm{fish})$. We found that behavioral response thresholds were elevated twofold to threefold for jittered sequences compared with natural sequences (single-sample $t$ test against $1 ; 1 \mathrm{~ms}$ jitter: $t_{(9)}=2.9, p=0.018 ; 3 \mathrm{~ms}$ jitter: $t_{(9)}=2.9$, $p=0.017$; 5 ms jitter: $t_{(9)}=3.6, p=0.0060$; Fig. $\left.2 D\right)$. These results reveal that fish are behaviorally sensitive to the precise timing of pulses within scallops.

\section{Single-neuron responses to scallops depend on interval selectivity}

The IPIs in electric communication signals are precisely encoded at the periphery and preserved by the ascending electrosensory system until activity reaches the midbrain ELp, where single neurons are sensitive to IPIs (for review, see Baker et al., 2013). To determine whether this population of IPI-selective neurons is sensitive to scallop temporal structure, we obtained in vivo intracellular whole-cell recordings from 56 ELp neurons during electrosensory stimulation with scallop stimuli (Fig. 3). IPI tuning was classified as high-pass ( $n=20$; Fig. $3 A)$, bandstop $(n=8$; Fig. $3 B)$, bandpass ( $n=7$; Fig. $3 C)$, low-pass $(n=16$; Fig. $3 D)$, complex $(n=3)$, or all-pass $(n=2)$, as described previously (Baker and Carlson, 2014).

Neurons responded differently to the same scallop sequences, depending on their IPI tuning (Fig. 3; Carlson, 2009). High-pass 
A (i)

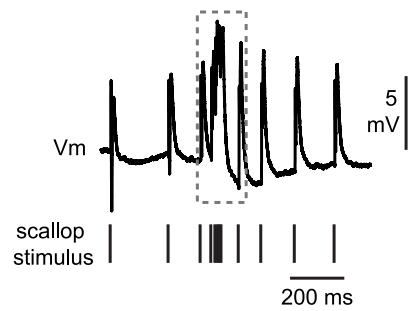

B (i)

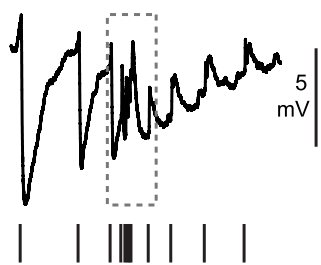

C (i)

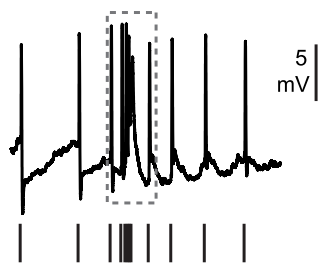

D (i)

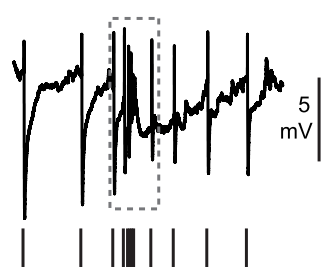

(ii)

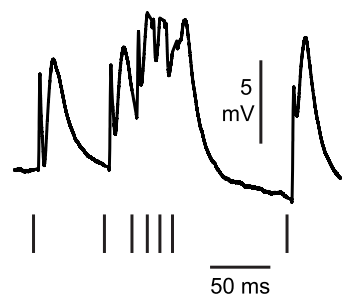

(ii)

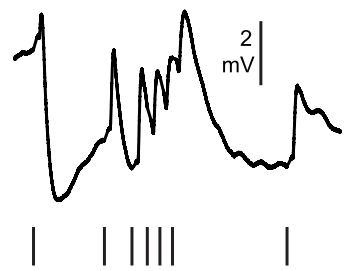

(ii)

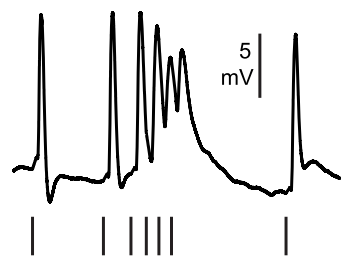

(ii)

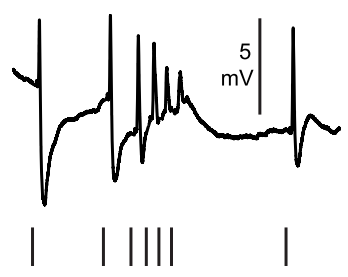

(iii)

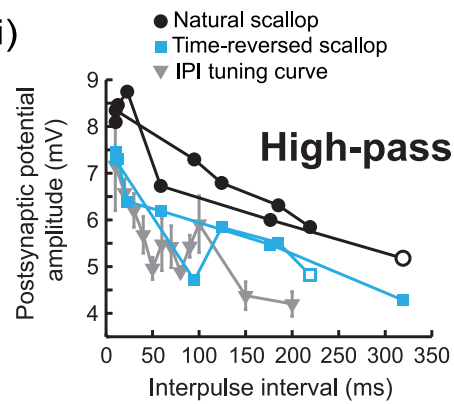

(iii)

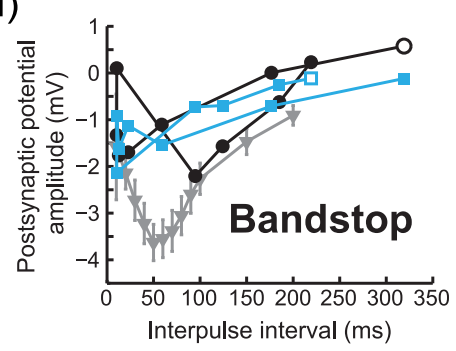

(iii)

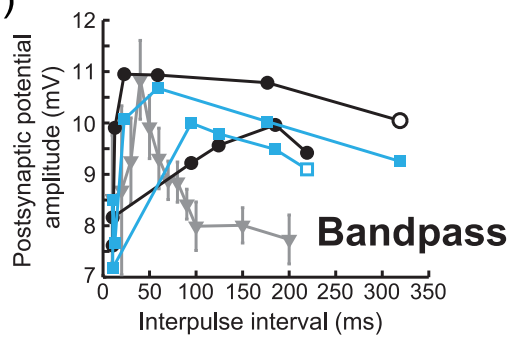

(iii)

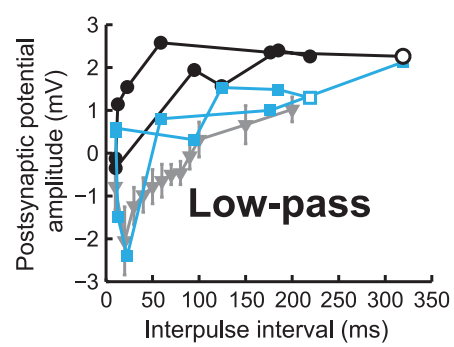

Figure 3. Single-neuron responses to scallops depend on interval selectivity. $A i$, Intracellular membrane potential $\left(V_{m}\right)$ recording from a high-pass neuron in response to a natural scallop ( $(M 3)$. The trace represents the membrane potential averaged over 10 stimulus presentations. Tick marks below the recording trace denote the time of each electric pulse in the stimulus. Aii, Close-up of the recording in Ai during the shortest IPIs in the scallop (dashed box in Ai). Aiii, PSP amplitudes in response to IPI tuning curve, natural scallop, and time-reversed scallop stimuli. The black curve reflects the responses shown in $\mathrm{Ai}$ and $\mathrm{Aii}$, and the blue curve represents the responses of the neuron to the time-reversed version of the same scallop. Open symbols indicate the response to the first IPI in the natural and time-reversed scallop stimuli. The gray curve represents the interval tuning curve of the neuron (see Materials and Methods). $\boldsymbol{B}-\boldsymbol{D}$, Same as $\boldsymbol{A}$ for a bandstop (B), bandpass ( $\boldsymbol{C}$, and low-pass (D) neuron during presentation of the same stimuli.

neurons produced the largest PSP amplitudes during the shortest IPIs in the scallop (Fig. 3A). Bandstop neurons produced the largest PSP amplitudes during the shortest and longest IPIs in the scallop (Fig. 3B). Bandpass neurons produced the greatest PSP amplitudes in response to intermediate IPIs in the scallop (Fig. $3 C)$. Low-pass neurons produced the largest PSP amplitudes in response to the longest IPIs of the scallop (Fig. 3D). In agreement with previous findings, the PSP amplitudes of some neurons depended on the direction of IPI change, such that response amplitudes to the same IPIs were slightly different for natural and time-reversed sequences of the same scallop (Fig. 3; Carlson, 2009). Thus, ELp neurons act as temporal filters for electric communication signals.
Subthreshold responses of interval-selective neurons are sensitive to changes in the sequence of intervals in scallops To determine whether ELp neurons are sensitive to the fine temporal structure of scallops, we recorded the intracellular responses of ELp neurons to natural, time-reversed, randomized, and jittered sequences of the same six scallops that we used in the behavior experiments. We used DFA to test whether responses to scallop manipulations were different enough to be quantitatively separated. For each neuron, we measured the PSP amplitudes evoked by each pulse on every stimulus presentation, and then used the PSP amplitudes in response to the second through the last pulse in DFA. We used responses to all six stimulus sequences (natural, time-reversed, randomized, and jitter of 1,3, and $5 \mathrm{~ms}$ ) 
A

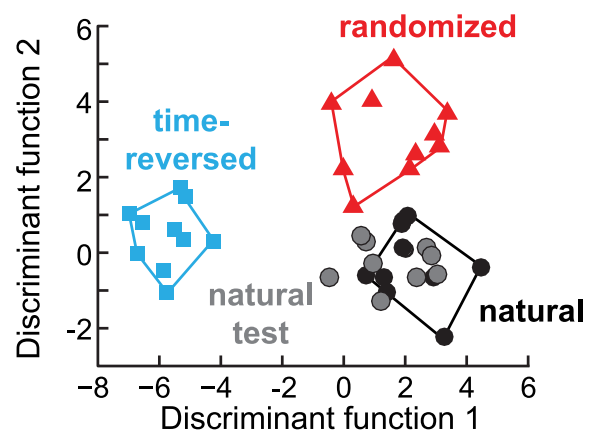

C

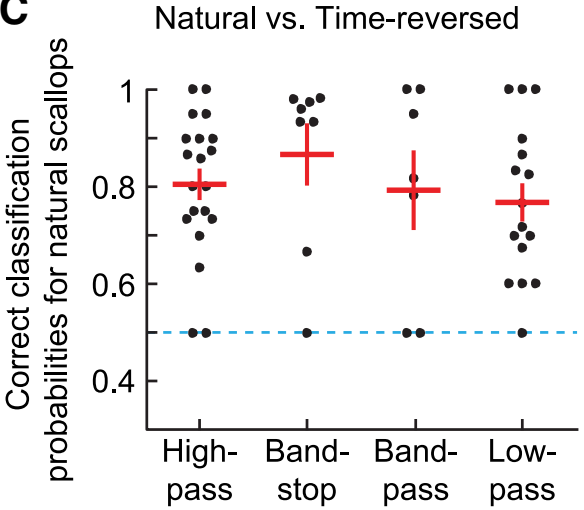

B

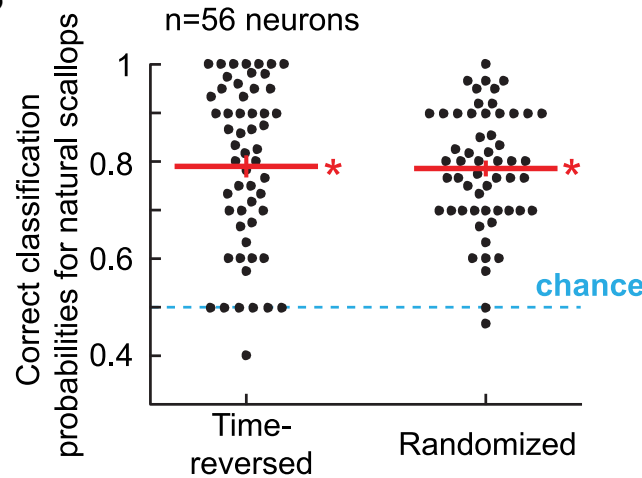

D

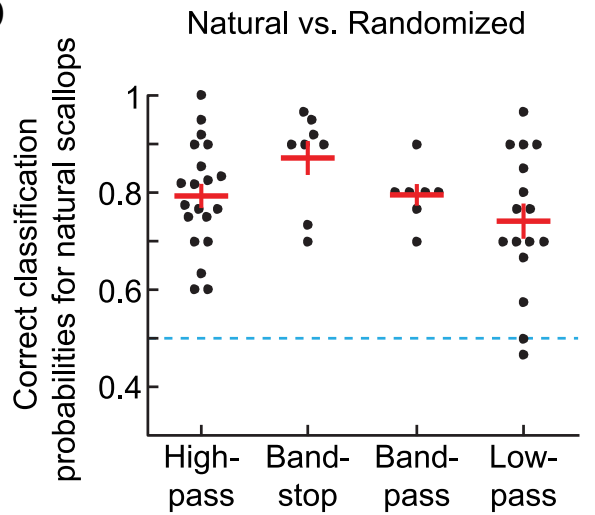

Figure 4. The responses of single neurons are sensitive to changes in the order of intervals in scallops. $\boldsymbol{A}$, DFA results for responses to natural, time-reversed, and randomized scallop (F2) sequences in a high-pass neuron. PSP amplitudes evoked by the second through last pulse in each of 10 stimulus presentations of natural, time-reversed, randomized, and jittered sequences were used to define the discriminant functions. We then used these functions to classify responses to 10 independent presentations of the natural scallop (gray circles) as natural or time-reversed sequences, and as natural or randomized sequences, based on Mahalanobis distances from group centroids. Each point reflects the responses of the neuron to one stimulus presentation. All 10 of the test responses of this neuron to the natural scallop were correctly classified as belonging to the natural sequence when compared with responses to the time-reversed sequence and with responses to the randomized sequence. $\boldsymbol{B}$, Probabilities of correct classification of natural scallop responses when compared with responses to time-reversed and randomized sequences for all neurons. *Single-sample $t$ test against chance (0.50), $p<0.000001$. C, Probability of correct classification of natural scallops compared with time-reversed scallops by IPI tuning. Complex ( $n=3$ neurons) and all-pass ( $n=2$ neurons) were excluded from this analysis. $\boldsymbol{D}$, Same as ( for natural vs randomized scallop sequences. Each point in $\boldsymbol{B}-\boldsymbol{D}$ represents the correct classification probability for a single neuron. If the neuron was presented with more than one natural scallop and corresponding manipulations, we used the classification probability averaged across natural scallops. Horizontal bars in $\boldsymbol{B}-\boldsymbol{D}$ represent the average across neurons, and error bars represent SEM.

to define discriminant functions that minimize intragroup variance while maximizing intergroup variance. We then used these functions to classify the responses of the neuron to 10 independent presentations of the natural scallop. If the responses to temporal manipulations were no different from those to natural scallops, the discriminant functions should correctly classify responses to natural scallops with chance performance.

To test whether subthreshold responses of individual neurons could be used to distinguish natural from time-reversed scallops, we used the discriminant functions to classify responses to each of 10 independent presentations of the natural scallop as either the natural or time-reversed sequence (Fig. 4A). Across all neurons, responses to natural scallops were correctly classified with a probability of $0.79 \pm 0.02$, which was significantly more frequent than that predicted by chance (single-sample $t$ test against 0.50 , $t_{(55)}=18, p<0.000001$; Fig. $4 B$ ). Correct classification probability for natural versus time-reversed scallops did not depend on IPI tuning (one-way ANOVA, $F_{(3)}=0.64, p=0.59$; Fig. $4 C$ ).

Next, we compared single-neuron responses to natural and randomized sequences. Responses to natural scallops were correctly classified with a mean probability of $0.79 \pm 0.02$, which was significantly more frequent than that predicted by chance (singlesample $t$ test against $0.50, t_{(55)}=13, p<0.000001$; Fig. $4 B$ ).
Classification performance for natural versus randomized sequences was independent of IPI tuning (one-way ANOVA, $F_{(3)}=$ 2.2, $p=0.097$; Fig. 4D). Collectively, these results demonstrate that the IPI selectivity of ELp neurons results in responses that are sensitive to the IPI sequence of natural temporal patterns.

\section{Subthreshold responses of interval-selective neurons are} sensitive to changes in the precise timing of pulses in scallops Next, we used the previously defined discriminant functions to classify responses to each of 10 independent presentations of natural scallops as natural or jittered sequences (Fig. 5A). In general, the responses of single ELp neurons could be used to correctly identify natural sequences significantly more frequently than predicted by chance (single-sample $t$ test against $0.50,1 \mathrm{~ms}$ jitter: $t_{(55)}=3.0, p=0.0044 ; 3 \mathrm{~ms}$ jitter: $t_{(55)}=4.2, p=0.00017 ; 5 \mathrm{~ms}$ jitter: $t_{(55)}=6.0, p<0.000001$; Fig. $\left.5 B\right)$.

Classification accuracy depended on the amount of added jitter (repeated-measures ANOVA, effect of jitter, $F_{(2)}=3.7$, $p=0.027$ ) but was independent of IPI tuning (repeatedmeasures ANOVA, effect of tuning, $F_{(3)}=0.13, p=0.95$; Fig. $5 C-E)$. There was no interaction effect between tuning and the amount of jitter $\left(F_{(6,96)}=0.28, p=0.94\right)$. These results demonstrate that the responses of single interval-selective neurons are 
A

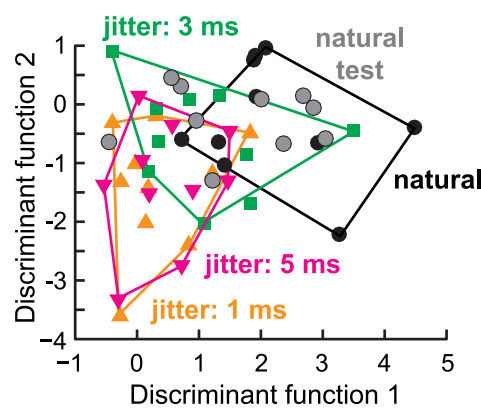

B

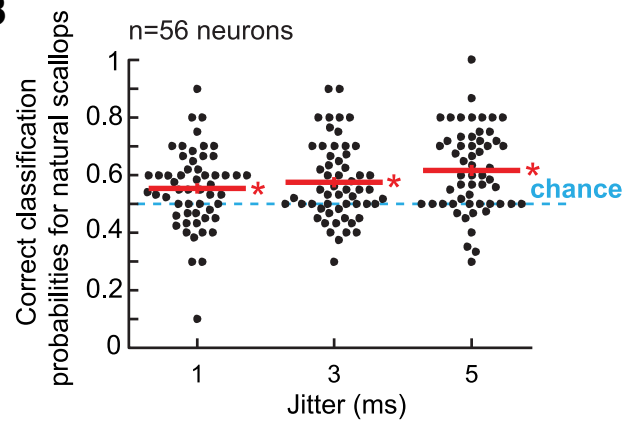

C

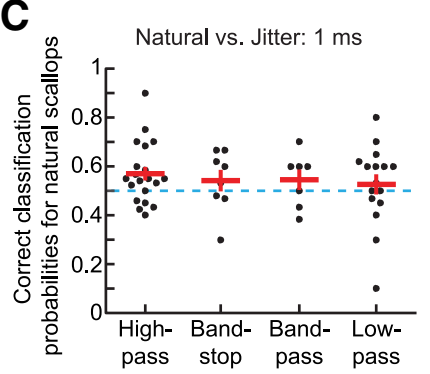

D

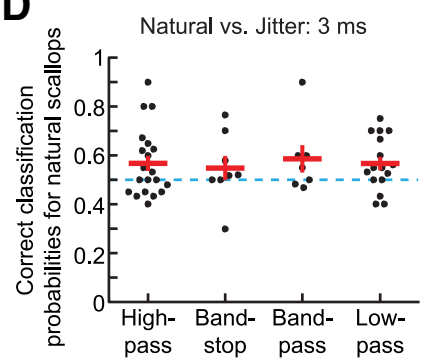

E

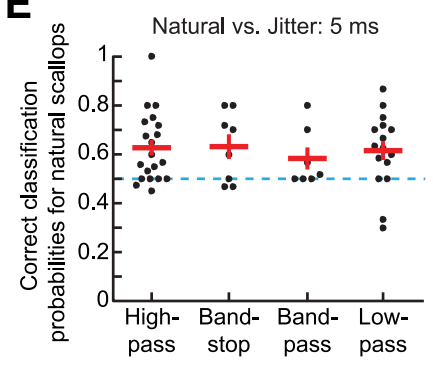

Figure 5. The responses of single neurons are sensitive to slight changes in the precise intervals in scallops. A, DFA results for responses to natural and jittered scallop (F2) sequences in a high-pass neuron (same neuron as in Fig. 4A). PSP amplitudes evoked by the second through the last pulse in each of 10 stimulus presentations of natural, time-reversed, randomized, and jittered sequences were used to define the discriminant functions. We then used these functions to classify responses to 10 additional presentations of the natural scallop (gray circles) as natural or jittered sequences based on Mahalanobis distances from group centroids. Seven, 4 , and 7 of 10 test responses of this neuron to the natural scallop were correctly classified when compared with sequences with 1, 3, and $5 \mathrm{~ms}$ of added jitter, respectively. Each point reflects the responses of the neuron to one stimulus presentation. $\boldsymbol{B}$, Correct classification probabilities for natural scallop responses compared with sequences with 1,3 , and $5 \mathrm{~ms}$ of added jitter for all neurons. * Single-sample $t$ test against chance $(0.50), p<0.01$. $\boldsymbol{C}-\boldsymbol{E}$, Probability of correct classification of natural vs $1 \mathrm{~ms}$ jittered sequences $(\boldsymbol{C})$, natural vs $3 \mathrm{~ms}$ jittered sequences $(\boldsymbol{D})$, and natural vs $5 \mathrm{~ms}$ jittered sequences $(\boldsymbol{E})$ by IPI tuning. $(\boldsymbol{C o m p l e x}$ ( $n=3$ neurons) and all-pass ( $n=2$ neurons) were excluded from this analysis. Each point in $\boldsymbol{B}-\boldsymbol{E}$ represents the correct classification probability for a single neuron. If the neuron was presented with more than one natural scallop and corresponding manipulations, we used the classification probability averaged across natural scallops. Horizontal bars represent the average across neurons, and error bars represent the SEM.

sensitive to slight changes in the precise intervals occurring in natural temporal patterns.

\section{Spiking responses reflect the sensitivity of subthreshold responses to scallop temporal structure}

We have shown that subthreshold PSP amplitudes in single neurons are sensitive to slight changes in natural scallop patterns. However, these subthreshold changes in membrane potential are not transmitted to downstream neurons; only spiking activity is. To investigate how spiking may affect the discriminability of single-neuron responses, we counted the number of spikes elicited by each pulse during every presentation of natural, timereversed, randomized, and jittered scallop sequences (Fig. 6A). Thirteen neurons produced spikes often enough to provide sufficient variation for classification by DFA. For each neuron, we used the spike counts in response to the second through the last pulse of natural, time-reversed, randomized, and jittered sequences to define the discriminant functions that maximized the separability of the responses to each manipulation. We then used these functions to classify responses to 10 independent presentations of the natural scallop.

On average, the responses of spiking neurons correctly classified natural compared with time-reversed and randomized scallops with a mean probability of $0.84 \pm 0.05$ and $0.77 \pm 0.05$, respectively, which were significantly more frequent than predicted by chance (single-sample $t$ test against 0.50, time-reversed: $t_{(12)}=6.9, p=0.000016$; randomized: $\left.t_{(12)}=5.0, p=0.00033\right)$. There was no difference in classification accuracy between spiking activity and subthreshold
PSPs for natural versus time-reversed scallops (paired $t$ test, $t_{(12)}=0.23, p=0.82$ ) or natural versus randomized scallops $\left(t_{(12)}=0.24, p=0.81\right.$; Fig. $\left.6 B\right)$.

Using spikes for DFA resulted in the correct classification of natural sequences with mean probabilities of $0.57 \pm 0.05$, $0.61 \pm 0.05$, and $0.55 \pm 0.06$ when compared with sequences with 1,3 , and 5 ms of added jitter, respectively, which were not significantly different from those predicted by chance (singlesample $t$ test against $0.50, t_{(12)}=0.056-1.3, p=0.21-0.96$ across all jitter values). Classification accuracy using spikes was not significantly different from that using PSPs for natural versus jittered sequences (repeated-measures ANOVA, effect of spikes vs PSPs, $F_{(1)}=1.0, p=0.33$; Fig. $6 C$ ). These results demonstrate that classification accuracy based on PSPs captures the classification accuracy of spiking activity that would be transmitted to downstream neurons.

A few neurons had correct classification probabilities that were well below chance (Fig. 6C). This result occurred when the responses to the natural and jittered stimuli were similar enough to be poorly separated by the discriminant functions. In these cases, the responses of the neuron were not reliable enough across stimulus presentations to be correctly classified.

\section{Pooling classification probabilities of multiple neurons improves discrimination performance}

We have shown that the responses of individual ELp neurons can be used to correctly classify natural versus time-reversed, randomized, and jittered scallop sequences. However, the electro- 

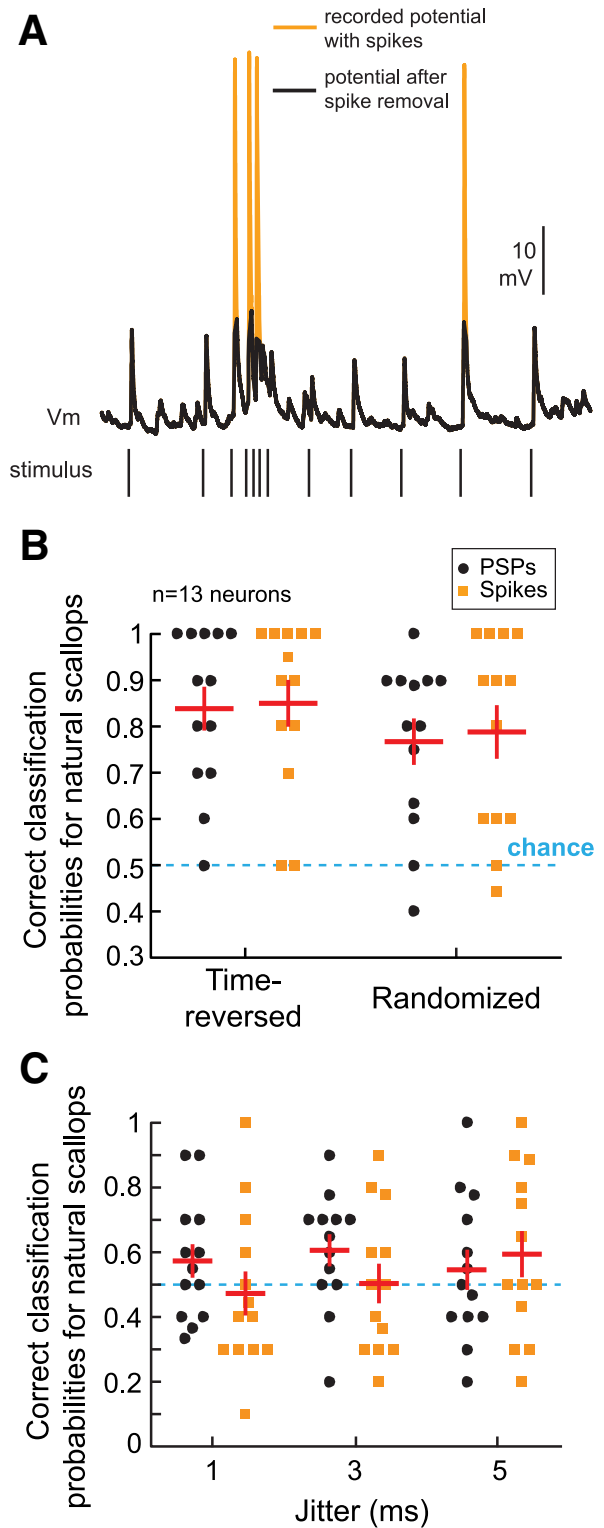

Figure 6. The discriminability of spiking responses reflects the discriminability of subthreshold PSPs. $A$, Intracellular recording of the membrane potential $\left(V_{m}\right)$ from a high-pass neuron in response to a natural scallop (F2). The recorded potential is shown in orange, and the potential after spike removal is shown in black. $\boldsymbol{B}$, Correct classification probabilities using PSP amplitudes (black) and spike counts (orange) for natural scallop responses compared with timereversed and randomized sequences. Each point reflects the correct classification probability for a single neuron. Horizontal bars represent the average across neurons, and error bars represent the SEM. C, Same as $\boldsymbol{B}$ for natural scallop responses compared with sequences with 1, 3, and 5 ms of added jitter.

sensory system does not have to rely solely on the output of any one ELp neuron to detect scallops. Rather, downstream neurons could integrate the output of multiple ELp neurons to identify temporally patterned communication signals. Therefore, we wanted to determine how classification performance would be affected if we pooled the responses of multiple ELp neurons together. We used Bayesian updating with single-neuron classification probabilities to estimate the probability of correct classification of natural versus time-reversed and randomized sequences for all possible combinations of two neurons and for 10,000 randomly selected combinations of 3-10 neurons. For neurons from which we recorded responses to multiple natural scallops and their manipulations, we used the single-neuron classification probabilities averaged over all scallops.

The probability of correct classification of natural versus time-reversed and randomized scallop sequences increased as the responses of more neurons were combined (Fig. 7A). Average correct classification probability reached 1 with groups of five neurons for both natural versus time-reversed and natural versus randomized scallops.

To determine how the IPI tuning of constituent neurons may affect classification, we compared the probability of correct classification of natural versus time-reversed and natural versus randomized scallops for groups consisting of all possible tuning compositions. In general, pairs of neurons from the same tuning class had classification probabilities for natural versus timereversed sequences that were significantly lower than pairs of neurons from different tuning classes (similar tuning, $0.91 \pm$ 0.01 ; dissimilar tuning, $0.93 \pm 0.003$; independent $t$ test: $t_{(1274)}=$ $-3.0, p=0.0026$ ). A similar pattern was observed for natural versus randomized scallops (similar tuning, $0.89 \pm 0.01$; dissimilar tuning, $0.93 \pm 0.002$; independent $t$ test: $t_{(1274)}=-6.5, p<$ 0.000001 ). Bandstop neurons formed pairs that tended to have the highest probability of correct classification (Fig. 7B). This finding is consistent with bandstop neurons tending to have greater correct classification probabilities than neurons of other tuning types (Fig. 4C,D). Bandstop neurons also tended to form groups of three and four neurons with higher classification probabilities than other combinations (Fig. 7C,D).

We also estimated the effect of pooling probabilities from groups of neurons on classification accuracy for natural versus jittered scallops (Fig. 8A). Combining classification probabilities of multiple neurons improved accuracy for natural sequences versus all tested amounts of added jitter (Fig. 8A). The relationships between correct classification probabilities and the number of neurons in a group were well fit with the functions, $y=0.12 *$ $\ln (x)+0.53\left(r^{2}=0.97\right)$ for natural versus $1 \mathrm{~ms}$ jittered sequences (Fig. $8 A$, orange); $y=0.15 * \ln (x)+0.55\left(r^{2}=0.98\right)$ for natural versus $3 \mathrm{~ms}$ jittered sequences (Fig. $8 A$, green); and $y=0.16 *$ $\ln (x)+0.61\left(r^{2}=1.0\right)$ for natural versus $5 \mathrm{~ms}$ jittered sequences (Fig. 8A, magenta). These equations predict that groups of 34, 15, and 9 neurons would be needed to achieve correct classification with probability of 0.95 for natural versus 1,3 , and 5 ms of jitteradded sequences, respectively.

To understand how the IPI tuning of constituent neurons may impact classification accuracy, we compared the probability of correct classification of all possible combinations of two to four neurons (Fig. 8B-D). IPI tuning affected classification performance differently for each amount of added jitter. For instance, pairs of neurons from dissimilar IPI tuning classes had significantly higher classification accuracies than pairs from the same tuning classes for natural versus $1 \mathrm{~ms}$ jittered sequences (dissimilar tuning, $0.59 \pm 0.01$; similar tuning, $0.55 \pm 0.02$; independent $t$ test: $\left.t_{(1274)}=-2.8, p=0.016\right)$. In contrast, for natural versus 3 $\mathrm{ms}$ jittered sequences, pairs with dissimilar IPI tuning had significantly lower classification accuracies than pairs from the same tuning classes (dissimilar tuning, $0.62 \pm 0.01$; similar tuning, $0.66 \pm 0.01$; independent $t$ test: $\left.t_{(1274)}=3.2, p=0.0014\right)$. There were no significant differences in the probability of correct classification for neuron pairs from the same versus different IPI tuning classes for natural versus $5 \mathrm{~ms}$ jittered sequences (dissimilar tuning, $0.71 \pm 0.01$; similar tuning, $0.69 \pm 0.01$; independent $t$ test: $\left.t_{(1274)}=-1.5, p=0.13\right)$. High-pass neurons tended to form groups with the highest correct classification probability for natural versus $1 \mathrm{~ms}$ jitter-added sequences, while low-pass neu- 
A

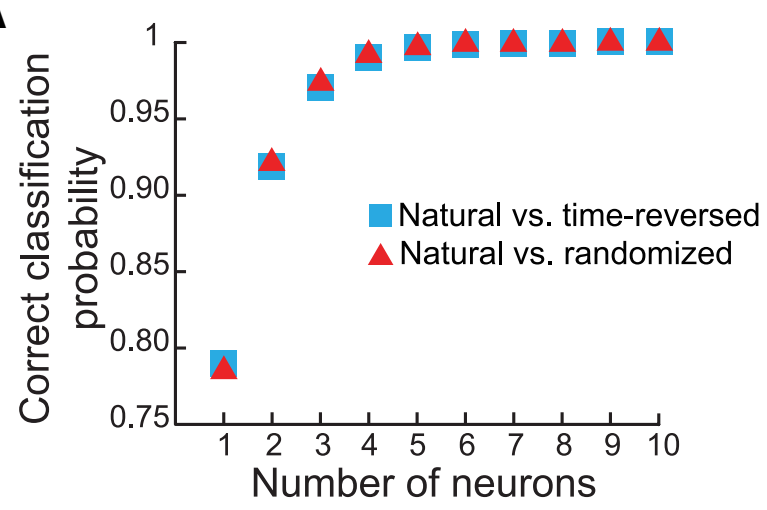

B
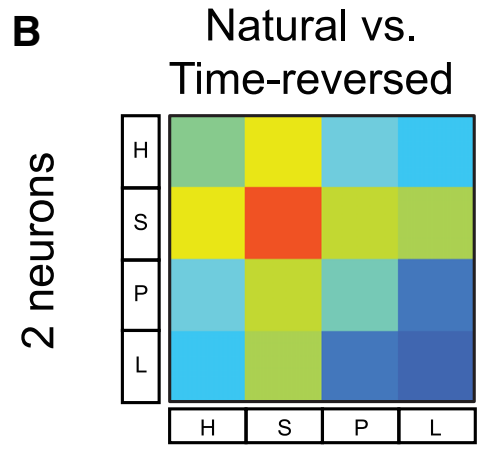

C
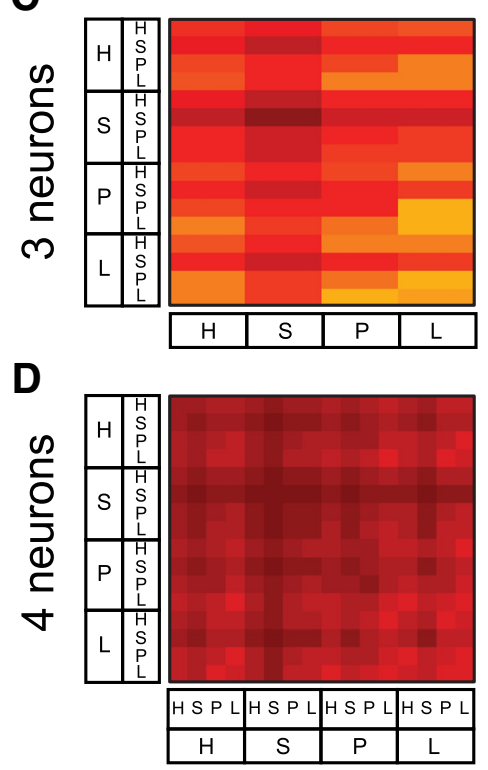

Natural vs. Randomized
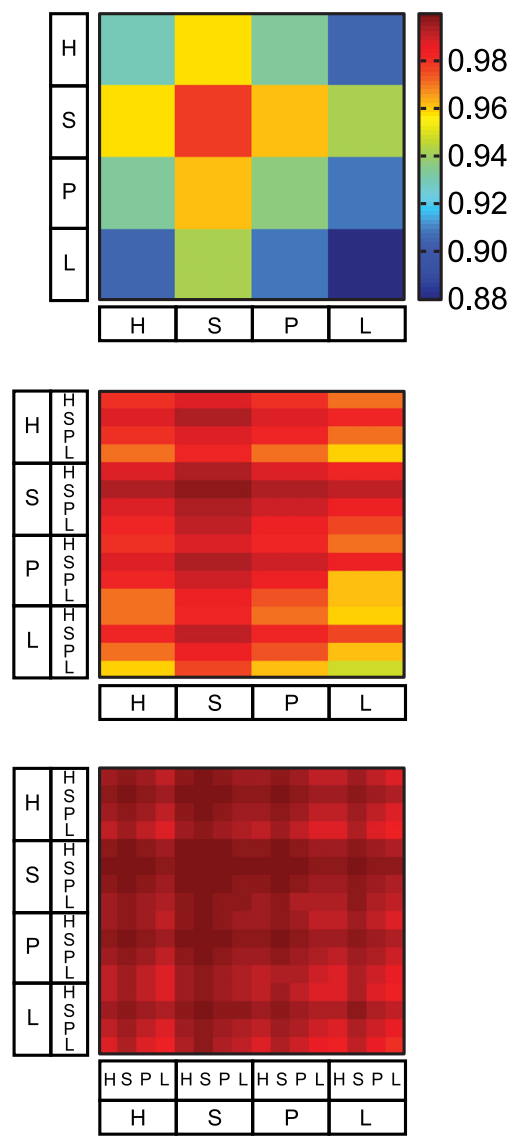

Figure 7. Pooling classification probabilities of multiple neurons improves discriminability of natural vs time-reversed and randomized sequences. $\boldsymbol{A}$, Probability of correct classification vs the number of neurons in a group for natural vs time-reversed (blue) and natural vs randomized (red) scallop sequences. We used Bayesian updating to estimate classification probabilities for all possible pairs of neurons and for 10,000 randomly selected combinations of 3 to 10 neurons from a total of 56 neurons. For neurons from which we recorded responses to more than one scallop, we used the single-neuron probabilities averaged across scallops. $\boldsymbol{B}$, Color map of classification accuracy of natural vs time-reversed (left) and natural vs randomized (right) scallop sequences for all possible pairs of neurons grouped by IPI tuning. We excluded three complex neurons and two all-pass neurons from these data. $\mathrm{H}$, high-pass; $S$, bandstop; $P$, bandpass; L, low-pass. $\boldsymbol{C}, \boldsymbol{D}$, Same as $\boldsymbol{B}$ for all possible groups of three $(\boldsymbol{C})$ and four $(\boldsymbol{D})$ neurons.

fication accuracy, while bandpass neurons tended to form groups with the lowest classification accuracy (Fig. 8D). Collectively, these results indicate that the IPI tuning of the pooled neurons can impact classification accuracy, and that the best combination of neurons for scallop pattern detection may be context-dependent.

\section{Responses of single interval-selective neurons are sensitive to individual variation in scallops}

Next we wanted to understand how single-neuron sensitivity to manipulated scallops related to sensitivity to natural variation in scallops. From 15 neurons, we collected responses to 10 repetitions of four natural scallops, each from a different individual. We used the PSP amplitudes evoked by the second through the last pulse in each scallop to define discriminant functions that maximally separated responses to the four scallops. We then used these functions to classify responses to 10 independent presentations of each of the same four natural scallops (Fig. 9A). On average, the probability of correct classification across neurons was $0.69 \pm 0.04$, which was significantly higher than that predicted by chance (single-sample $t$ test against $0.25, t_{(14)}=$ $10.5, p<0.000001)$. There were no significant differences in correct classification probability across the four scallops (repeated-measures ANOVA: $F_{(3)}=2.0$, $p=0.12$; Fig. $9 B$ ).

Single-neuron correct classification probabilities for scallops of different individuals were positively correlated with those for natural versus randomized scallops (Spearman's $r=0.52, t_{(13)}=2.2$, $p=0.045)$. In contrast, there was no relationship between single-neuron correct classification probability for scallops of different individuals and those for natural versus time-reversed (Spearman's $r=$ $\left.0.22, t_{(13)}=0.78, p=0.44\right)$ or those for natural versus jittered scallops (Spearman's $r=-0.13$ to $0.22, t_{(13)}=-0.49$ to $0.81, p=0.43-0.65$ across all jitter values). Therefore, responses that were good at discriminating natural from randomized scallops were also good at discriminating among scallops from different individuals.

To understand how the number of scallops the responses were used to dis-

rons tended to form groups with the lowest classification accuracy (Fig. $8 B$ ). Bandpass neurons tended to form the bestperforming groups for natural versus $3 \mathrm{~ms}$ jittered sequences, while bandstop neurons tended to form the worst-performing groups (Fig. 8C). For natural versus $5 \mathrm{~ms}$ jittered sequences, bandstop neurons tended to form groups with the highest classi- criminate among might affect classification accuracy, we computed single-neuron classification accuracies for all combinations of two scallops and three scallops (Fig. 9C). Classification accuracy decreased when responses were used to discriminate among a larger number of scallops, but accuracy remained above chance performance (Fig. 9C). 
A

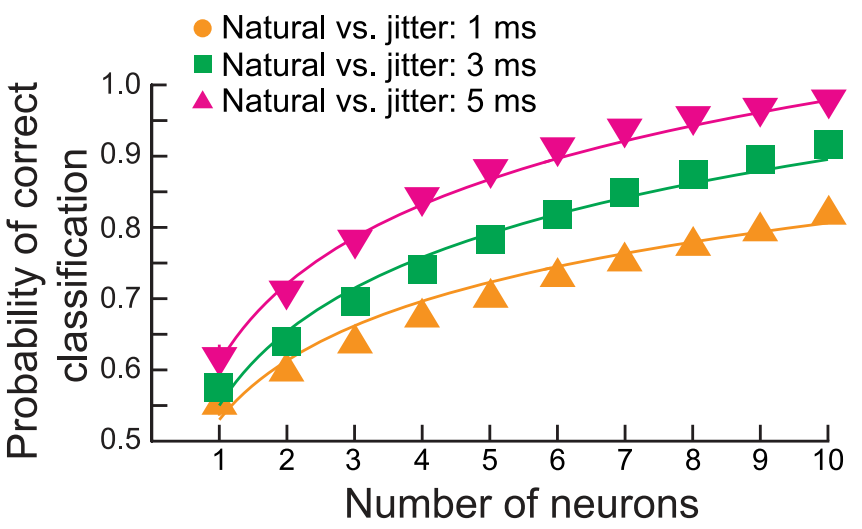

B
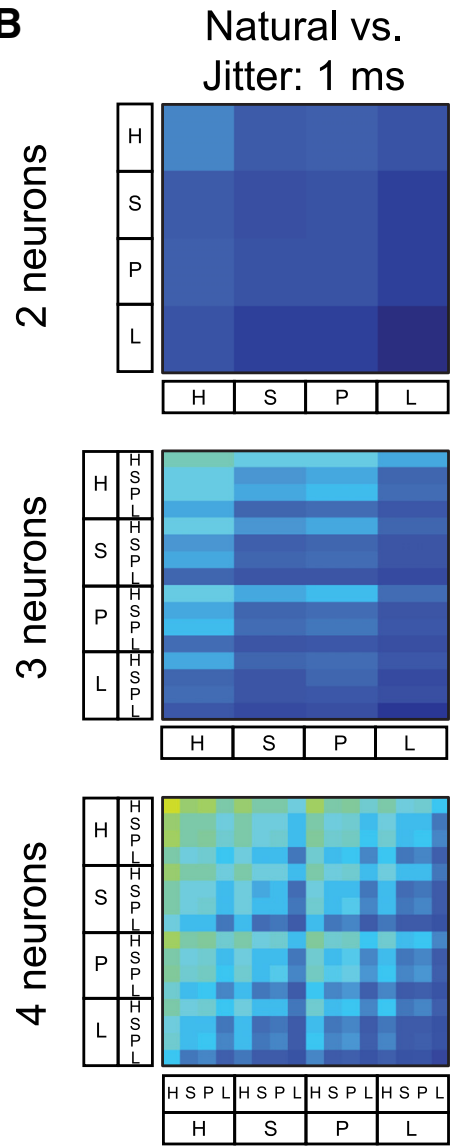

C Natural vs. Jitter: $3 \mathrm{~ms}$
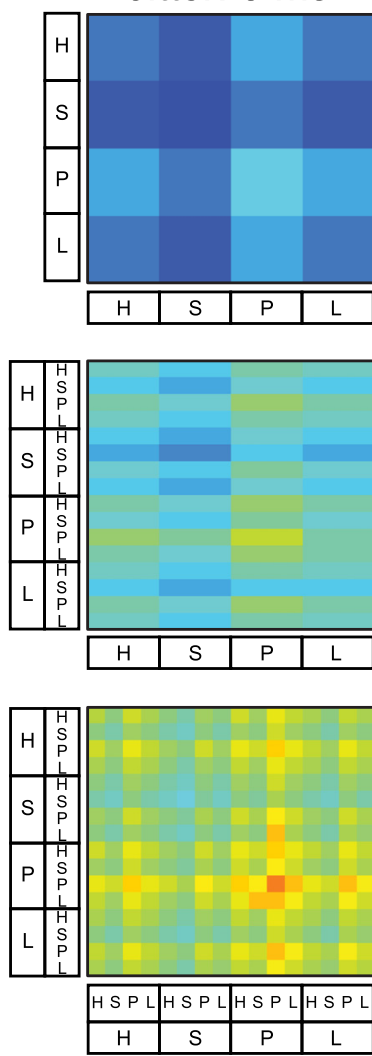

D Natural vs. Jitter: $5 \mathrm{~ms}$
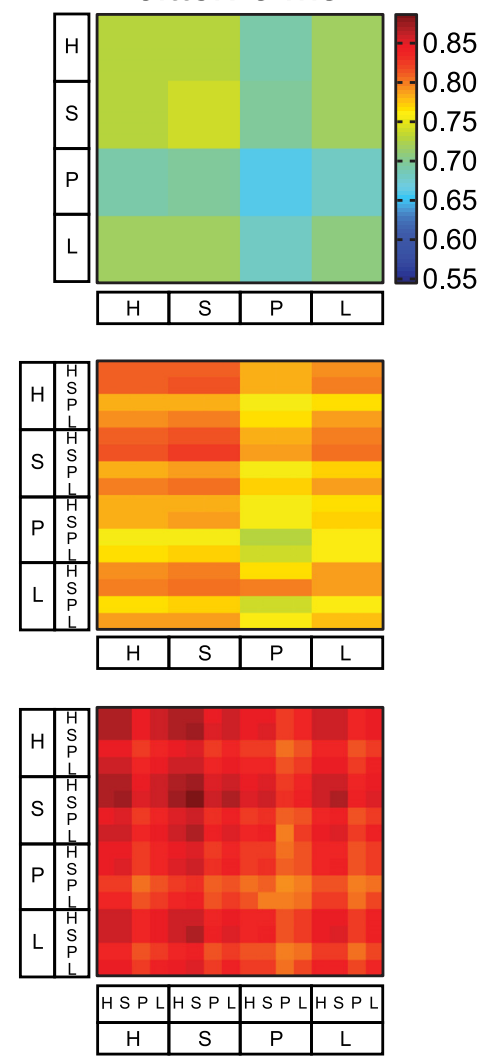

Figure 8. Pooling classification probabilities of multiple neurons improves discriminability of natural sequences versus sequences with added jitter. $\boldsymbol{A}$, Probability of correct classification vs the number of neurons in a group for natural vs 1 ms jitter-added (orange), natural vs 3 ms jitter-added (green), and natural vs 5 ms jitter-added (magenta) scallop sequences. We used Bayesian updating to estimate classification probabilities for all possible pairs and for 10,000 randomly selected combinations of $3-10$ neurons from a total of 56 neurons. For neurons from which we recorded responses to more than one scallop, we used the single-neuron probabilities averaged across scallops. The orange line represents the function $y=0.12 * \ln (x)+0.53\left(r^{2}=0.97\right)$; the green line represents the function $y=0.15 * \ln (x)+0.55\left(r^{2}=0.98\right)$; and the magenta line represents the function $y=0.16 * \ln (x)+0.61\left(r^{2}=0.99\right)$. $B$, Color map of classification accuracy of natural vs 1 ms jitter-added scallop sequences for all possible combinations of two (top), three (middle), and four (bottom) neurons. We excluded three complex neurons and two all-pass neurons from these data. H, high-pass; S, bandstop; P, bandpass; L, low-pass. C, D, Same as $\boldsymbol{B}$ for natural vs 3 ms jitter-added sequences ( $\boldsymbol{D}$ ) and for natural vs 5 ms jitter-added sequences ( $\boldsymbol{E}$ ).

Pooling the responses of multiple neurons enhanced the discriminability of natural scallops (Fig. 9D). Groups of two, three, and four neurons reached a correct classification probability of 0.95 for discriminating among two, three, and four scallops, respectively (Fig. 9D). These data suggest a 1:1 relationship between the number of scallops to discriminate among and the number of neurons required for $95 \%$ classification accuracy. Thus, the output of ELp neurons contains enough information to resolve natural scallop variation.
To understand how natural variation in scallop patterns may compare to the manipulations we studied, we computed jitter values between the four natural scallops with the same number of IPIs. For a pair of scallops, we measured the SD of the difference in times of corresponding EODs in each sequence, with the time of the first EOD defined as $0 \mathrm{~ms}$. We also computed the jitter between all six natural scallops and their respective timereversed sequences, as well as 100,000 randomly chosen randomized sequences. In all cases, jitter between sequences was 
A

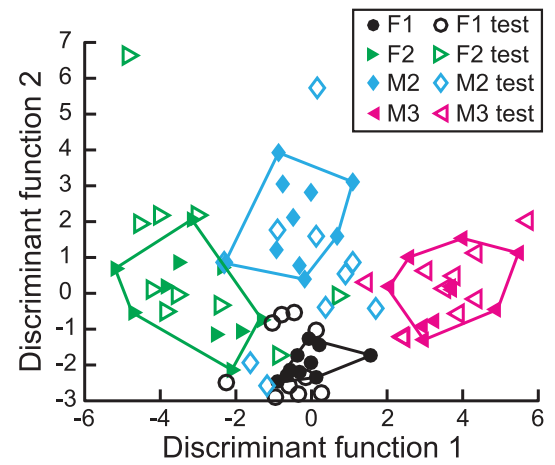

B

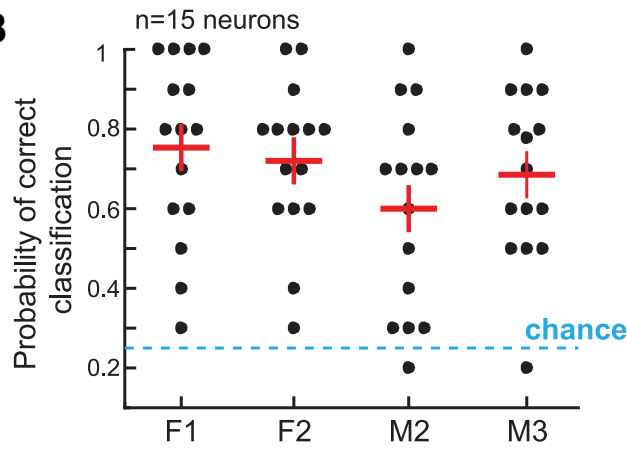

C

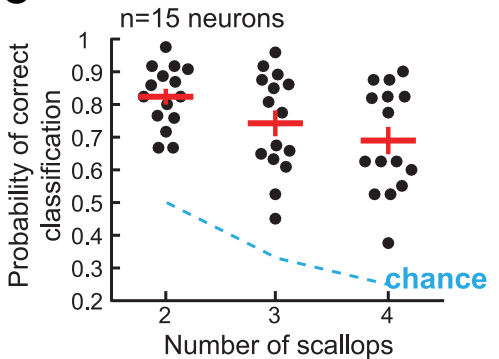

D

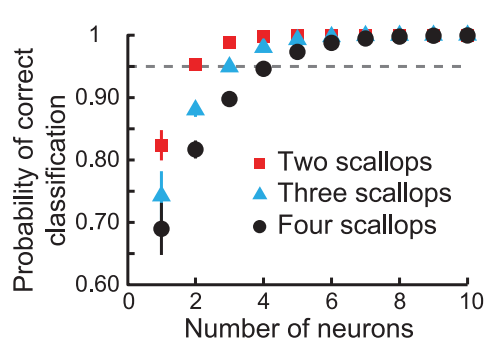

E

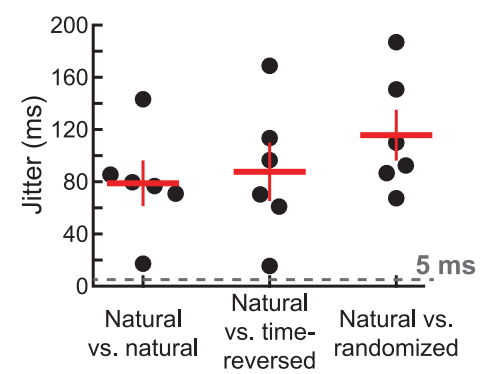

Figure 9. The responses of single neurons are sensitive to individual variation in scallops. A, DFA results for responses to natural scallop sequences from four different individuals (F1, F2, M2, M3) in a high-pass neuron. PSP amplitudes evoked by the second through the last pulse in each of 10 stimulus presentations of the four scallops were used to define the discriminant functions. We then used these functions to classify responses to 10 additional presentations of each natural scallop (open symbols) as one of the four natural scallops based on Mahalanobis distances from group centroids. All 10 repetitions of the $F 1$ and $M 3$ scallops were correctly classified. Eight of 10 and 4 of 10 repetitions of the $F 2$ and $M 2$ scallops, respectively, were correctly classified. Each point reflects the responses of the neuron to one stimulus presentation. $\boldsymbol{B}$, Probabilities of correct classification by scallop. Each point represents the probability for one neuron. Horizontal bars represent the average across neurons, and error bars represent the SEM. C, Probability of correct classification vs the number of scallops available to discriminate among. Each point represents the probability for one neuron averaged across all combinations of two scallops ( $n=6$ possible combinations), three scallops ( $n=4$ possible combinations), and four scallops ( $n=1$ possible combination). Horizontal bars represent the average across neurons, and error bars represent the SEM. D, Probability of correct classification vs the number of neurons in a group for all possible combinations of two (red), three (blue), and four (black) natural scallops. We used Bayesian updating to estimate classification probabilities for all possible combinations of single-neuron probabilities from the responses of 15 neurons to the same four scallops. Each point represents the correct classification probability averaged across all possible combinations of neurons and scallops. $E$, Mean $j$ itter values between the four natural scallops with the same number of IPIs, and between all six natural scallops and their respective time-reversed and 100,000 randomly selected randomized sequences. To estimate jitter, we calculated the SD of the difference in the time of corresponding EODs in each pair of stimuli. Each point represents the jitter between a pair of sequences. Horizontal bars represent the average across pairs of stimuli, and error bars represent the SEM.

larger than the 1,3 , and $5 \mathrm{~ms}$ jittered stimuli that we tested (Fig. $9 E$ ). That the responses of single neurons are sensitive to timing changes well below comparable measurements of natural variation underscores the degree to which ELp neurons preserve the temporal information in electric communication displays.

\section{Discussion}

Our results demonstrate that behavioral sensitivity to millisecond timing variations in natural scallops is due at least in part to a population of midbrain neurons that serve as temporal filters for electric communication signals. Both behavioral and singleneuron responses were sensitive to changes in the order of IPIs, as well as to the addition of as little as $1 \mathrm{~ms}$ of jitter to natural scallops. Remarkably, there was enough information present in single-neuron responses to distinguish among scallops produced by different individuals. Collectively, these results illustrate how a population of interval-selective neurons can contribute to the detection of variation in behaviorally relevant temporal patterns of presynaptic input.

Temporal patterns of spikes encode information in a wide range of sensory and motor circuits (Mauk and Buonomano, 2004). However, the decoding of precise temporal sequences is not well understood. In most model systems, it is difficult to precisely control the temporal pattern of inputs onto temporally selective neurons in vivo. Here we overcame this limitation using mormyrid electric fish, in which the temporal patterns of presynaptic inputs onto interval-selective central neurons match the temporal patterns in electrosensory stimuli (for review, see Baker et al., 2013). Furthermore, electric communication signals can easily be manipulated in behaviorally relevant ways. This means that not only do we have direct control over the temporal patterns of presynaptic input onto interval-selective neurons, but we can also record the responses of these neurons to input patterns that would occur during natural communication behavior. This system allowed us to quantify the ability of single neurons and populations of neurons to detect variation in precise temporal sequences of sensory input. Other studies have investigated neural coding of natural versus time-reversed auditory communication signals in a variety of animals (Margoliash, 1983; van Stokkum, 1987; Doupe and Konishi, 1991; Esser et al., 1997; Gehr et al., 2000; Lu et al., 2001; Suta et al., 2008), but the spectrotemporal structure of these signals is much more complex than the series of electric pulses used by mormyrids. Since the electrosensory stimulus itself is essentially a spike train whose temporal patterns are preserved centrally, our results provide insight into neural coding and decoding in addition to communication signal feature detection.

We found that adding as little as $1 \mathrm{~ms}$ of jitter to natural scallop patterns increased behavioral response thresholds, despite the fact 
that scallops from different individuals vary by much $>1 \mathrm{~ms}$ of jitter. It is important to note that behavioral response thresholds are not simple readouts of signal detection. Instead, fish must detect the signal, analyze the temporal pattern, and "decide" whether and how to respond. This processing will be affected by stimulus salience and motivation. Thus, even though natural scallops vary across individuals, response thresholds are more sensitive to natural patterns than artificial patterns. We have shown here that the first stage of temporal filtering in the electrosensory system preserves enough information to inform the recognition of a scallop from a non-scallop. It would be interesting to test the limit of behavioral sensitivity by determining the smallest change to the scallop that would affect response thresholds.

The ability of single neurons to provide enough information to discriminate among scallops from different individuals is in line with previous findings in auditory and electrosensory pathways. The spiking responses of songbird field L neurons, grasshopper auditory receptors and higher-order neurons, and wave-type electric gymnotiform fish (which evolved their electric sense independently of mormyrids) hindbrain neurons can be used to discriminate among conspecific signals produced by different individuals (Machens et al., 2003; Wang et al., 2007; Ronacher et al., 2008; Marsat and Maler, 2010). However, here we go beyond describing single-neuron variability in response to natural signal variation to quantify the ability of single neurons to distinguish among precise temporal manipulations.

ELp neurons exhibit a great deal of diversity in responses to IPIs. We classify these responses into six categories (i.e., highpass, low-pass, bandpass, bandstop, complex, and all-pass) based on quantitative measures of PSP amplitudes evoked by IPIs (Carlson, 2009; George et al., 2011; Baker and Carlson, 2014). However, even within these classes, responses are variable regarding parameters such as bandwidth, best IPI, and sensitivity to the direction of IPI change (Carlson, 2009; George et al., 2011; Ma et al., 2013; Baker and Carlson, 2014; Kohashi and Carlson, 2014). This wide variety of response properties arises from a combination of synaptic mechanisms, including temporal summation and short-term depression, as well as intrinsic membrane properties (George et al., 2011; Baker and Carlson, 2014; Kohashi and Carlson, 2014). Pooling the classification probabilities of multiple neurons improved discrimination performance, with the amount of improvement depending on IPI tuning. Several studies have reported that a population of neurons with heterogeneous response properties can result in better discrimination performance than a population of neurons with homogeneous response properties (Shamir and Sompolinsky, 2006; Chelaru and Dragoi, 2008; Osborne et al., 2008; Holmstrom et al., 2010; Marsat and Maler, 2010; but see Schneider and Woolley, 2010). However, a combination of heterogeneity and homogeneity in olfactory mitral bulb cells provided the most benefits to stimulus encoding, and the optimal combination of heterogeneous versus homogeneous neural responses depended on the stimulus (Tripathy et al., 2013). The tuning combinations resulting in the greatest discrimination performance varied across the tested scallop manipulations, indicating that the best combination of ELp response properties may also be stimulusdependent.

The improvement in classification probability by pooling responses over many neurons suggests that downstream neurons could integrate the output of multiple ELp neurons to achieve high discriminability between natural temporal patterns. Our method of pooling probabilities considered the responses of each neuron with equal weights. However, downstream neurons could achieve even better performance than that predicted here by adjusting synaptic weights accordingly (Shamir and Sompolinsky, 2006; Barbour et al., 2007). For instance, a neuron receiving one input with high classification accuracy and another input with low classification accuracy could assign a stronger weight to the better performing input. One such mechanism that could facilitate changes in synaptic weights is spike timing-dependent plasticity (Feldman, 2012). Indeed, the timing of ELp responses, which we did not analyze here, might provide additional information for downstream neurons to discriminate natural from jittered scallops. ELp neurons project to the isthmic granule nucleus, inferior olive, subpreminential nucleus, and medioventral nucleus (Baker et al., 2013). However, how these regions respond to communication signals and the possible convergence patterns of ELp inputs are unknown.

Although ELp output contains enough information to resolve interindividual scallop variation, whether fish use this information remains to be determined. The individually stereotyped EOD waveform contains identifying information such as species, sex, and dominance status, and can even be used to identify individuals in some species (Graff and Kramer, 1992; Friedman and Hopkins, 1996; Carlson et al., 2000; Carlson, 2002; Hanika and Kramer, 2005). Combining the stereotyped EOD waveform with individually characteristic scallop patterns could facilitate individual recognition. Another potential function of stereotyped scallop patterns could be to signal information related to fitness, such as size, health, fecundity, and/or fighting ability. Since scallops have been hypothesized to play a role as an aggressive advertisement or territorial display signal (Carlson and Hopkins, 2004), these individually distinctive temporal patterns could be used to "size up" potential competitors.

Here we explored how a population of neurons encodes one particular electric communication signal. However, mormyrids produce at least three additional types of temporal patterns during social encounters, called accelerations, rasps, and creaks, which all consist of a larger number of IPIs than scallops (Carlson and Hopkins, 2004; Wong and Hopkins, 2007). The ability of single ELp neurons to distinguish between individual scallops consisting of only 10 IPIs suggests that these neurons would likely also be able to distinguish between different classes of communication signals, as well as variation within these classes (Carlson and Hopkins, 2004). Furthermore, neurons with different IPI tuning properties respond differently to scallops, accelerations, and rasps (Carlson, 2009). Since ELp neurons are involved in detecting more than just scallop sequences, it is possible that neurons whose responses poorly discriminated scallop variation may instead be better suited to discriminate among other communication signals. Since we measured responses to scallops in isolation, it remains to be determined how scallop encoding and recognition may be affected by preceding temporal patterns during ongoing signaling. It will be necessary to investigate population coding of additional communication displays, as well as to assess the coding and discrimination performance of downstream neurons, to gain a fuller understanding of how interval-selective neurons in ELp mediate the detection of the full range of behaviorally relevant temporal patterns in communication signals.

\section{References}

Baker CA, Carlson BA (2014) Short-term depression, temporal summation, and onset inhibition shape interval tuning in midbrain neurons. J Neurosci 34:14272-14287. CrossRef Medline 
Baker CA, Kohashi T, Lyons-Warren AM, Ma X, Carlson BA (2013) Multiplexed temporal coding of electric communication signals in mormyrid fishes. J Exp Biol 216:2365-2379. CrossRef Medline

Barbour B, Brunel N, Hakim V, Nadal JP (2007) What can we learn from synaptic weight distributions? Trends Neurosci 30:622-629. CrossRef Medline

Bell CC, Grant K (1989) Corollary discharge inhibition and preservation of temporal information in a sensory nucleus of mormyrid electric fish. J Neurosci 9:1029-1044. Medline

Bennett MV (1965) Electroreceptors in mormyrids. Cold Spring Harb Symp Quant Biol 30:245-262. CrossRef Medline

Carlson BA (2002) Electric signaling behavior and the mechanisms of electric organ discharge production in mormyrid fish. J Physiol Paris 96: 405-419. CrossRef Medline

Carlson BA (2009) Temporal-pattern recognition by single neurons in a sensory pathway devoted to social communication behavior. J Neurosci 29:9417-9428. CrossRef Medline

Carlson BA, Hopkins CD (2004) Stereotyped temporal patterns in electrical communication. Anim Behav 68:867-878. CrossRef

Carlson BA, Kawasaki M (2006) Stimulus selectivity is enhanced by voltagedependent conductances in combination-sensitive neurons. J Neurophysiol 96:3362-3377. CrossRef Medline

Carlson BA, Hopkins CD, Thomas P (2000) Androgen correlates of socially induced changes in the electric organ discharge waveform of a mormyrid fish. Horm Behav 38:177-186. CrossRef Medline

Carlson BA, Hasan SM, Hollmann M, Miller DB, Harmon LJ, Arnegard ME (2011) Brain evolution triggers increased diversification of electric fishes. Science 332:583-586. CrossRef Medline

Chelaru MI, Dragoi V (2008) Efficient coding in heterogeneous neuronal populations. Proc Natl Acad Sci U S A 105:16344-16349. CrossRef Medline

Cooley WW, Lohnes PR (1971) Multivariate data analysis. New York: Wiley.

Doupe AJ, Konishi M (1991) Song-selective auditory circuits in the vocal control system of the zebra finch. Proc Natl Acad Sci U S A 88:1133911343. CrossRef Medline

Edwards CJ, Alder TB, Rose GJ (2002) Auditory midbrain neurons that count. Nat Neurosci 5:934-936. CrossRef Medline

Esser KH, Condon CJ, Suga N, Kanwal JS (1997) Syntax processing by auditory cortical neurons in the FM-FM area of the mustached bat Pteronotus parnellii. Proc Natl Acad Sci U S A 94:14019-14024. CrossRef Medline

Ewing AW, Bennet-Clark HC (1968) The courtship songs of Drosophila. Behaviour 31:288-301. CrossRef

Feldman DE (2012) The spike-timing dependence of plasticity. Neuron 75: 556-571. CrossRef Medline

Fortune ES, Rose GJ (1997a) Temporal filtering properties of ampullary electrosensory neurons in the torus semicircularis of Eigenmannia: evolutionary and computational implications. Brain Behav Evol 49:312-323. Medline

Fortune ES, Rose GJ (1997b) Passive and active membrane properties contribute to the temporal filtering properties of midbrain neurons in vivo. J Neurosci 17:3815-3825. Medline

Fortune ES, Rose GJ (2000) Short-term synaptic plasticity contributes to the temporal filtering of electrosensory information. J Neurosci 20:71227130. Medline

Fortune ES, Rose GJ (2003) Voltage-gated Na + channels enhance the temporal filtering properties of electrosensory neurons in the torus. J Neurophysiol 90:924-929. CrossRef Medline

Friedman MA, Hopkins CD (1996) Tracking individual mormyrid electric fish in the field using electric organ discharge waveforms. Anim Behav 51:391-407. CrossRef

Gehr DD, Komiya H, Eggermont JJ (2000) Neuronal responses in cat primary auditory cortex to natural and altered species-specific calls. Hear Res 150:27-42. CrossRef Medline

George AA, Lyons-Warren AM, Ma X, Carlson BA (2011) A diversity of synaptic filters are created by temporal summation of excitation and inhibition. J Neurosci 31:14721-14734. CrossRef Medline

Gittelman JX, Li N, Pollak GD (2009) Mechanisms underlying directional selectivity for frequency-modulated sweeps in the inferior colliculus revealed by in vivo whole-cell recordings. J Neurosci 29:13030-13041. CrossRef Medline

Goel A, Buonomano DV (2014) Timing as an intrinsic property of neural networks: evidence from in vivo and in vitro experiments. Philos Trans $R$ Soc Lond B Biol Sci 369:20120460. CrossRef Medline
Graff C, Kramer B (1992) Trained weakly-electric fishes Pollimyrus isidori and Gnathonemus petersii (Mormyridae, Teleostei) discriminate between waveforms of electric pulse discharges. Ethology 90:279-292.

Grothe B (1994) Interaction of excitation and inhibition in processing of pure-tone and amplitude-modulated stimuli in the medial superior olive of the moustached bat. J Neurophysiol 71:706-721. Medline

Hanika S, Kramer B (2005) Intra-male variability of its communication signal in the weakly electric fish, Marcusenius macrolepidotus (South African form), and possible functions. Behaviour 142:145-166. CrossRef

Holmstrom LA, Eeuwes LB, Roberts PD, Portfors CV (2010) Efficient encoding of vocalizations in the auditory midbrain. J Neurosci 30:802-819. CrossRef Medline

Kohashi T, Carlson BA (2014) A fast BK-type KCa current acts as a postsynaptic modulator of temporal selectivity for communication signals. Front Cell Neurosci 8:286. CrossRef Medline

Lloyd JE (1966) Studies on the flash communication system in Photinus fireflies. Ann Arbor, MI: University of Michigan Miscellaneous Publications 130:1-95.

Loftus-Hills JJ, Littlejohn MJ (1971) Pulse repetition rate as basis for mating call discrimination by two sympatric species of Hyla. Copeia 1971: 154-156. CrossRef

Lu T, Liang L, Wang X (2001) Temporal and rate representations of timevarying signals in the auditory cortex of awake primates. Nat Neurosci 4:1131-1138. CrossRef Medline

Lyons-Warren AM, Hollmann M, Carlson BA (2012) Sensory receptor diversity establishes a peripheral population code for stimulus duration at low intensities. J Exp Biol 215:2586-2600. CrossRef Medline

Lyons-Warren AM, Kohashi T, Mennerick S, Carlson BA (2013) Retrograde fluorescent labeling allows for targeted extracellular single-unit recording from identified neurons in vivo. J Vis Exp 76:e3921. CrossRef

Ma X, Kohashi T, Carlson BA (2013) Extensive excitatory network interactions shape temporal processing of communication signals in a model sensory system. J Neurophysiol 110:456-469. CrossRef Medline

Machens CK, Schütze H, Franz A, Kolesnikova O, Stemmler MB, Ronacher B, Herz AV (2003) Single auditory neurons rapidly discriminate conspecific communication signals. Nat Neurosci 6:341342. CrossRef Medline

Margoliash D (1983) Acoustic parameters underlying the responses of song-specific neurons in the white-crowned sparrow. J Neurosci 3:1039 1057. Medline

Marsat G, Maler L (2010) Neural heterogeneity and efficient population codes for communication signals. J Neurophysiol 104:2543-2555. CrossRef Medline

Mauk MD, Buonomano DV (2004) The neural basis of temporal processing. Annu Rev Neurosci 27:307-340. CrossRef Medline

Osborne LC, Palmer SE, Lisberger SG, Bialek W (2008) The neural basis for combinatorial coding in a cortical population response. J Neurosci 28: 13522-13531. CrossRef Medline

Pluta SR, Kawasaki M (2010) Temporal selectivity in midbrain electrosensory neurons identified by modal variation in active sensing. J Neurophysiol 104:498-507. CrossRef Medline

Pollack G (2000) Who, what, where? Recognition and localization of acoustic signals by insects. Curr Opin Neurobiol 10:763-767. CrossRef Medline

Ronacher B, Wohlgemuth S, Vogel A, Krahe R (2008) Discrimination of acoustic communication signals by grasshoppers (Chorthippus biguttulus): temporal resolution, temporal integration, and the impact of intrinsic noise. J Comp Psychol 122:252-263. CrossRef Medline

Rose G, Capranica RR (1983) Temporal selectivity in the central auditory system of the leopard frog. Science 219:1087-1089. CrossRef Medline

Rose GJ, Fortune ES (1996) New techniques for making whole-cell recordings from CNS neurons in vivo. Neurosci Res 26:89-94. Medline

Rose GJ, Leary CJ, Edwards CJ (2011) Interval-counting neurons in the anuran auditory midbrain: factors underlying diversity of interval tuning. J Comp Physiol A Neuroethol Sens Neural Behav Physiol 197:97-108. CrossRef Medline

Sakai M, Chimoto S, Qin L, Sato Y (2009) Neural mechanisms of interstimulus interval-dependent responses in the primary auditory cortex of awake cats. BMC Neurosci 10:10. CrossRef Medline

Schneider DM, Woolley SM (2010) Discrimination of communication vocalizations by single neurons and groups of neurons in the auditory midbrain. J Neurophysiol 103:3248-3265. CrossRef Medline 
Serrier J, Moller P (1989) Patterns of electric organ discharge activity in the weakly electric fish Brienomyrus niger L. (Mormyridae). J Exp Biol 48:235-244.

Shamir M, Sompolinsky H (2006) Implications of neuronal diversity on population coding. Neural Comput 18:1951-1986. CrossRef Medline

Suta D, Popelár J, Syka J (2008) Coding of communication calls in the subcortical and cortical structures of the auditory system. Physiol Res 57 [Suppl 3]:S149-S159. Medline

Tripathy SJ, Padmanabhan K, Gerkin RC, Urban NN (2013) Intermediate intrinsic diversity enhances neural population coding. Proc Natl Acad Sci U S A 110:8248-8253. CrossRef Medline

Trussell LO (1999) Synaptic mechanisms for coding timing in auditory neurons. Annu Rev Physiol 61:477-496. CrossRef Medline van Stokkum IH (1987) Sensitivity of neurons in the dorsal medullary nucleus of the grassfrog to spectral and temporal characteristics of sound. Hear Res 29:223-235. CrossRef Medline

Wang L, Narayan R, Graña G, Shamir M, Sen K (2007) Cortical discrimination of complex natural stimuli: can single neurons match behavior? J Neurosci 27:582-589. CrossRef Medline

Wong RY, Hopkins CD (2007) Electrical and behavioral courtship displays in the mormyrid fish Brienomyrus brachyistius. J Exp Biol 210:22442252. CrossRef Medline

Xu-Friedman MA, Hopkins CD (1999) Central mechanisms of temporal analysis in the knollenorgan pathway of mormyrid electric fish. J Exp Biol 202:1311-1318. Medline 